\title{
CONSTRUCCIÓN DE UNA CARTA \\ ARQUEOLÓGICA DE LA LOCALIDAD \\ ARQUEOLÓGICA PUNTA MEDANOSA \\ (PATAGONIA ARGENTINA) \\ MEDIANTE HERRAMIENTAS SIG
}

\section{CONSTRUCTION OF AN ARCHAEOLOGICAL MAP OF PUNTA MEDANOSA ARCHAEOLOGICAL LOCALITY (ARGENTINE PATAGONIA) USING GIS TOOLS}

\author{
Miguel Ángel Zubimendi \\ Recibido: 05/04/2021 - Aceptado: 01/07/2021 \\ DOI: https://dx.doi.org/10.5944/etfi.14.2021.30598
}

\begin{abstract}
Resumen
En este trabajo se presenta una sistematización de datos georreferenciados obtenidos a lo largo de 30 años de estudios en la localidad arqueológica Punta Medanosa (Provincia de Santa Cruz, Patagonia Argentina). A partir del ordenamiento de la información generada en distintos momentos y con diferentes objetivos dentro de un mismo proyecto de investigación, se pretende dar cuenta de las características y la riqueza arqueológica de esta localidad, que se destaca dentro de la costa patagónica continental por su extensión y alta densidad de contextos arqueológicos, en especial concheros y diferentes modalidades de entierro a lo largo del Holoceno medio y tardío. Se empleó Sistemas de Información Geográfica y se presenta una carta arqueológica, como producto que puede ser empleado no sólo para el análisis académico, sino también para la gestión del patrimonio arqueológico, dado que presenta un creciente grado de alteración antrópica en los últimos años.
\end{abstract}

Palabras clave

Punta Medanosa; Sistema de Información Geográfica; Carta arqueológica; Gestión

\section{Abstract}

In this paper we present a systematization of georeferenced data obtained over 30 years of studies in the archaeological locality of Punta Medanosa, located on the north coast of Santa Cruz (Argentine Patagonia). This locality has a great quantity of

1. CONICET. Unidad Académica Caleta Olivia, Universidad Nacional de la Patagonia Austral; División Arqueología del Museo de La Plata, Facultad de Ciencias Naturales y Museo de la Universidad Nacional de La Plata. Paseo del Bosque s/n. La Plata, Argentina. C. P. 1900. Argentina, mikelzubimendi@gmail.com 
shell middens and different burial modalities of middle and late Holocene. From the organization of information generated at different times and with different objectives within the same research project, it is intended to account for the characteristics and archaeological richness of Punta Medanosa, which stands out within the continental Patagonian coast for its extension and high density of archaeological sites. In this way, a database was created using Geographic Information Systems in which, the existing data were entered, considering the variability of archaeological and contemporary contexts, and a series of archaeological charts and thematic cartographies are presented. We hope that these products can be used for the analysis and management of the archaeological heritage of this locality, especially considering the high degree of anthropic alteration that is suffering.

\section{Keywords}

Punta Medanosa; Geographical Information System; Archaeological map; management 


\section{INTRODUCCIÓN}

En las últimas décadas se comprueba en Argentina un marcado crecimiento del uso de bases de datos arqueológicas empleando Sistemas de Información Geográfica (SIG), tanto a nivel nacional (ver, por ejemplo, De Feo et al. 20I8; Figuerero Torres e Izeta 20II, entre otros), como en la región patagónica (entre otros, Vitores 20I5; Magnin 20I7). Estas herramientas, entre otros usos, posibilitan sistematizar una gran cantidad de datos no sólo para generar cartografías o análisis espaciales estadísticos, sino también para la gestión de patrimonio cultural (Lanzelotti 20I7; Mariano et al. 20I4; Panizza y Oliva 20I8; Pastor et al. 2013).

En la Patagonia, se destaca la presentación de una carta arqueológica de la cuenca media del Limay (Homar y Guillermo 20I7; Vitores 2015) a partir de la información generada durante décadas en el marco de rescates y diversos proyectos de investigación. Otro antecedente es el desarrollo de una base de datos utilizando SIG en el Parque Nacional Bosques Petrificados de Jaramillo realizado por Magnin (2017) a partir de la sistematización de la información de datos georreferenciados existentes en un periodo que abarca más de 20 años de estudios sistemáticos.

El presente trabajo se enmarca dentro de esta tendencia, de la cual existen distintos ejemplos de la aplicación y uso de herramientas SIG en el marco de proyectos de investigación arqueológica (por ejemplo, Assandri y Gastaldi 2018; Lanzelloti 2020; Lanzelotti et al. 2015; Magnin 20I7; Panizza y Oliva 2018; Vitores 2015). Estos forman parte de un proceso de migración de registros en papel y su integración con datos más recientemente tomados mediante GPS, hacia aquellos basados en sistemas de computación mediante la aplicación de SIG, ya que permiten colectar, manejar, integrar, visualizar y analizar información geográficamente referenciada (Conolly 2008). El objetivo es organizar los datos generados durante las últimas décadas en la localidad arqueológica Punta Medanosa, la cual, por sus características particulares como alta densidad de sitios concheros y concentraciones de entierros humanos y presencia de instrumentos poco comunes (arpones de hueso, por ejemplo), permiten afirmar que se destaca dentro de la arqueología de la costa patagónica (Castro et al. 200I, 2004, 20II; Zangrando 20I8; Zubimendi 2019a). De esta forma, se conformó un corpus de información que puede ser útil para sectorizar los espacios en esta localidad y generar un estado de situación presentado en forma de carta arqueológica. La cual puede ser especialmente útil para la definición de futuras líneas de acción, tanto para la investigación como para la protección y extensión del patrimonio cultural (Martín Ruiz et al. 1995-1996).

Si bien los primeros estudios realizados en Punta Medanosa dentro del marco del proyecto Arqueología de la Costa Norte de Santa Cruz se iniciaron a fines de la década de I980, recién en el año I999 se comenzaron a levantar datos georreferenciados con posicionadores satelitales que son los que se presentan y se utilizan mayormente en este trabajo. Dado que a lo largo de los años se han registrado gran cantidad de datos, se decidió integrar, ordenar y actualizar la información existente para poder obtener una imagen general de la arqueología de esta localidad mediante la construcción de una carta arqueológica. En esta, se presenta toda la información espacial disponible, a partir de la cual se procede a analizar la distribución de tipos 
de sitios arqueológicos y otros contextos antrópicos, como restos de naufragios o pircados recientes. Posteriormente, se postula y analiza una diferenciación espacial intra-localidad según las características del registro arqueológico. Por último, se propone una zonificación considerando el registro arqueológico tal como se presenta actualmente, y teniendo en cuenta las alteraciones antrópicas recientes identificadas, su intensidad y características, con el fin de colaborar en una planificación adecuada de la posible gestión del patrimonio por parte de las instituciones gubernamentales que deben protegerlo.

\section{LA LOCALIDAD ARQUEOLÓGICA PUNTA MEDANOSA}

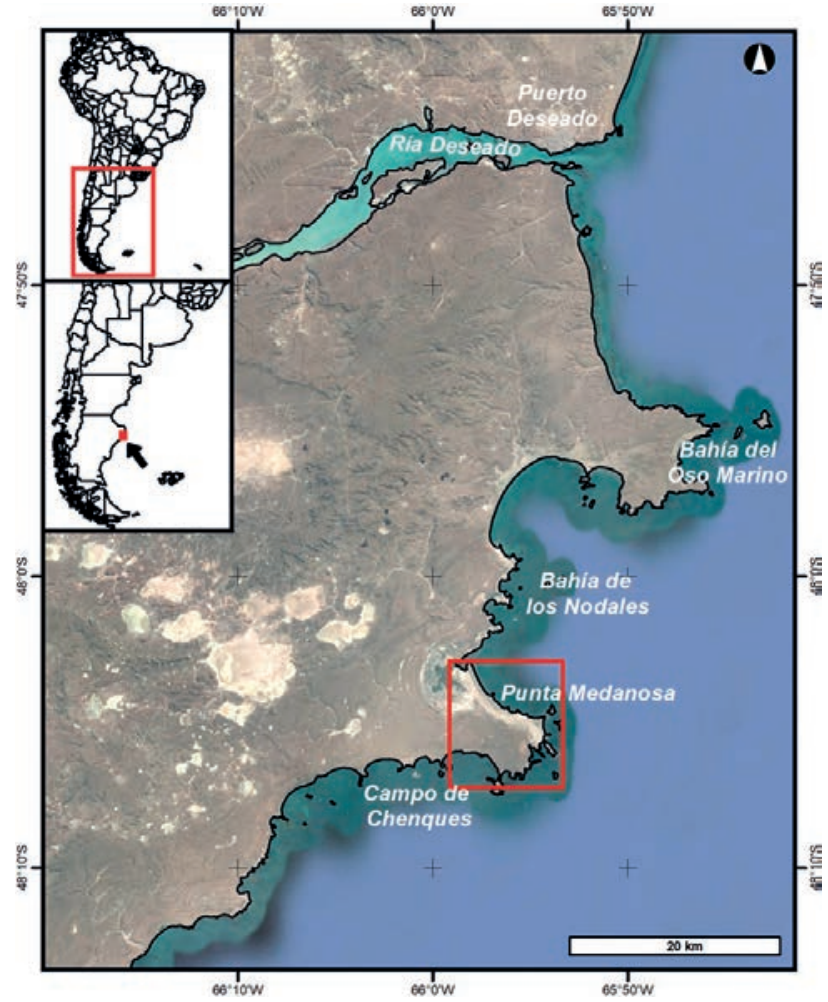

FIGURA 1. UBICACIÓN DE LA LOCALIDAD ARQUEOLÓGICA PUNTA MEDANOSA
El recorte espacial del presente trabajo corresponde a la denominada Punta Medanosa, pequeña península que mide aproximadamente $7 \mathrm{~km}$ de largo por $4 \mathrm{~km}$ de ancho. Se ubica en la costa central de la Patagonia argentina, en la costa norte de la provincia de Santa Cruz (Castro et al. 2003), sector sur de la ría Deseado (Zubimendi et al. 2015; Figura I). Este accidente geográfico se halla a aproximadamente $40 \mathrm{~km}$ al sur en línea recta de la ciudad de Puerto Deseado y constituye el límite sur de la bahía de los Nodales. Abarca todo el litoral y su interior inmediato desde la ensenada Ferrer hasta cerca del sitio Campo de Chenques ${ }^{2}$. Se encuentra dentro de área del Proyecto Arqueología de la Costa Norte de Santa Cruz, dirigido desde mediados de la década de I980 por la Dra. A. Castro, y se han realizado estudios en diversas oportunidades desde entonces.

\subsection{CARACTERÍSTICAS AMBIENTALES E HISTORIA}

La punta Medanosa propiamente dicha se extiende en sentido este-oeste, y se encuentra parcialmente separada del continente por un sistema de marismas

2. Este último, donde se encuentra una gran cantidad de estructuras de entierro de tipo chenque (ver Castro y Moreno 2000; Castro et al. 2001; Zilio, Hammond y Castro 2017), no queda abarcado dentro del presente trabajo por hallarse por fuera del sector de península de la Punta Medanosa. 

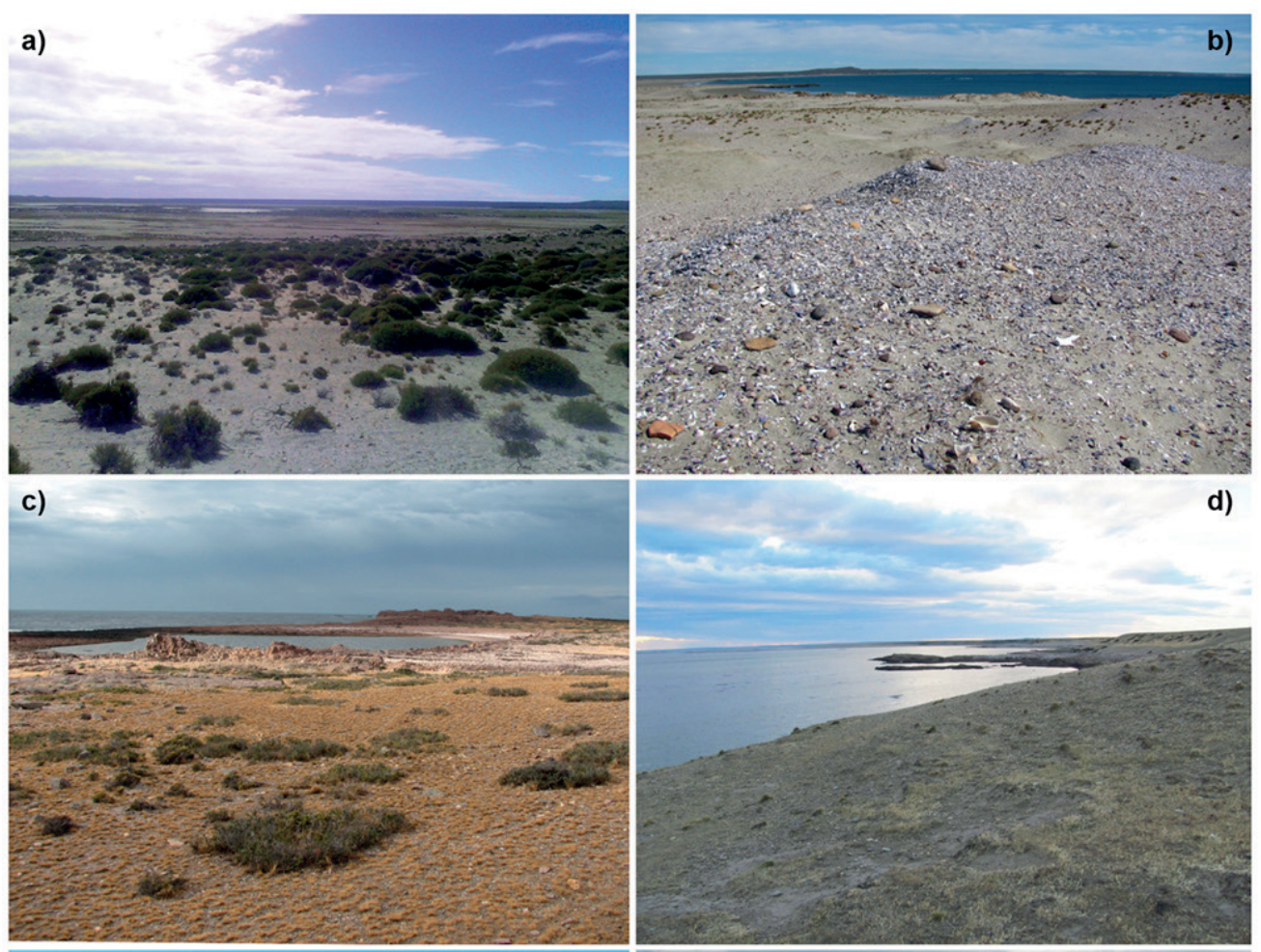

e)

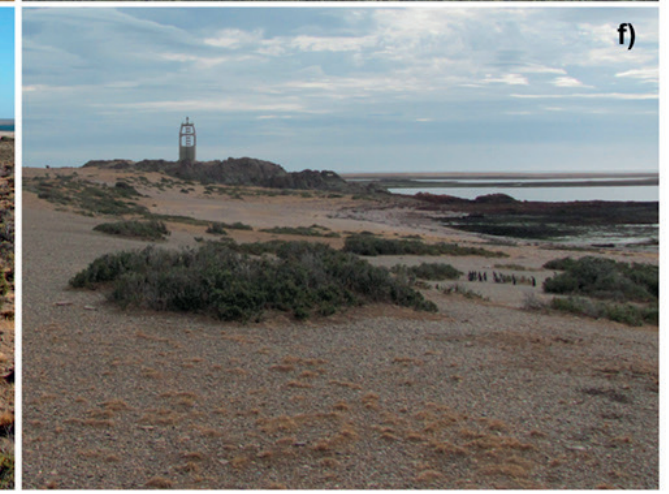

FIGURA 2. VISTAS DE DISTINTAS ZONAS DE PUNTA MEDANOSA. REFERENCIAS: A) ENSENADA FERRER, AL FRENTE SITIOS CONCHEROS SOBRE DUNAS PARCIALMENTE VEGETADAS, Y FONDO EL SISTEMA DE MARISMAS; B) PUNTA BUQUE, AL FRENTE CONCHERO EXTENSO Y DE ALTA DENSIDAD, AL FONDO SE OBSERVAN DUNAS EN EROSIÓN CON CONCHEROS INSERTOS EN LA MATRIZ EÓLICA; C) COSTA SUDESTE; D) COSTA SUDOESTE, AL FRENTE MESETA, Y AL FONDO ACANTILADOS Y AFLORAMIENTOS ROCOSOS COSTEROS; E) MESETA ALTA, AL FONDO DE OBSERVAN CORDONES LITORALES CON DUNAS EN EROSIÓN Y CONCHEROS; Y F) BALIZA, A LA DERECHA SE OBSERVA UN PIÑO DE PINGÜINOS DE MAGALLANES. LAS UBICACIONES DE LAS FOTOS DE EJEMPLO SE HALLAN EN LA FIGURA 8. Figura elaboración propia

(denominado ensenada Ferrer), que conforman un conjunto de cursos meandrosos que se inundan con las mareas altas normales (Figura 2.a). La punta está formada por una combinación de rocas volcánicas (pórfidos) y sedimentitas marinas y terrestres (Feruglio I950; Panza et al. I994). Los pórfidos actúan como roca de base aflorando especialmente en el extremo este y sur. La parte norte está conformada por cordones litorales subparalelos a la línea de costa actual. Hacia el mar, se diferencian depósitos de playa que constituyen varias terrazas marinas holocénicas. Los cordones 
tienen una altura máxima de entre 7 y in metros sobre el nivel del mar actual y han sido formados con posterioridad a la ingresión marina del Holoceno medio (Constante 20or; Medina et al. 20I4; Zubimendi 20I9a). En ellos se observa un muy escaso desarrollo edáfico y vegetacional, aunque en muchos casos se encuentran ocultos por extensas acumulaciones medanosas y mantos de arena, en su mayoría apoyados sobre las paleoplayas. Las acumulaciones de dunas, junto a los fuertes y abundantes vientos, le otorgan al paisaje una gran dinámica (Figura 2.b). A su vez, en la actualidad, es una zona visitada frecuentemente por turistas, por lo que presenta un gran impacto antrópico moderno (Zubimendi 20I9b). En la costa sudeste es donde los afloramientos rocosos se presentan expuestos en la línea costera, conformando en algunos casos acantilados activos e inactivos, así como plataformas de abrasión ascendidas. Se observan también cordones litorales de rodados expuestos o edafizados (Figura 2.c). En la costa sudoeste, donde la meseta alta se acerca a la costa, predominan los acantilados de hasta $20 \mathrm{msnm}$, con pequeños cañadones colgantes y algunos afloramientos rocosos (Figura 2.d). Por último, la meseta alta ocupa la mayor parte de Punta Medanosa, y se caracteriza por presentar una superficie plana, levemente ondulada (Figura 2.e).

En su aspecto ecológico, Punta Medanosa pertenece al matorral xerófilo mixto del Golfo San Jorge y al ambiente de la meseta central. La fauna se encuentra representada principalmente por una gran colonia de reproducción de pingüinos de Magallanes (Spheniscus magellanicus; Schiavini et al. 2005). Esta cuenta con un número de entre 14.000 y 22.000 parejas reproductivas y abarca un área de aproximadamente 34,5 ha. Los nidos se encuentran mayormente excavados en un sustrato arcillo-arenoso o bajo grandes matas de molle (Schinus poligamus) (Cruz 2007). También existen otras colonias otras menores de cormoranes (Phalacrocorax sp.), y bancos de moluscos (principalmente Mytilus edulis y Nacella magellanica). En el pasado existieron grandes loberías, tanto en la punta como en los islotes adyacentes (Carrara 1952; Schiavini et al. 1999). La fauna terrestre más abundante consiste en choiques (Pteronemia pennata), maras (Dolichotis patagonica) y guanacos (Lama guanicoe).

La punta Medanosa, como accidente geográfico, es conocido desde el siglo XVII, cuando fue descubierto por los hermanos Nodales a comienzos de enero de I6I9, quienes la denominaron Santa María. Estos navegantes recorrieron la zona, y destacaron la gran cantidad de lobos y aves marinas (García del Nodal y García del Nodal I62I: I4-23). El nombre original no prosperó y durante el siglo XIX, en la cartografía comienza a ser registrado como Hilly Point (Groussac I9I2: 40I), denominación que se mantiene hasta mediados del siglo XX (Figura 3.a b y c). Recién en dicho siglo se la comienza a denominar Punta Medanosa (Figura 3.d), nombre que probablemente derive a partir de una traducción inexacta del inglés en el marco de una política de uniformizar y priorizar la nomenclatura en español (Paz Soldán I884). A su vez, los pobladores de las ciudades cercanas llaman a la parte norte de este accidente geográfico Punta Buque, denominación que, si bien no es oficial, está muy extendida.

Desde la década de igio se encuentra dentro de la estancia El Amanecer, cuyos dueños actuales son la familia Vidal. Durante más de un siglo se ha practicado de 


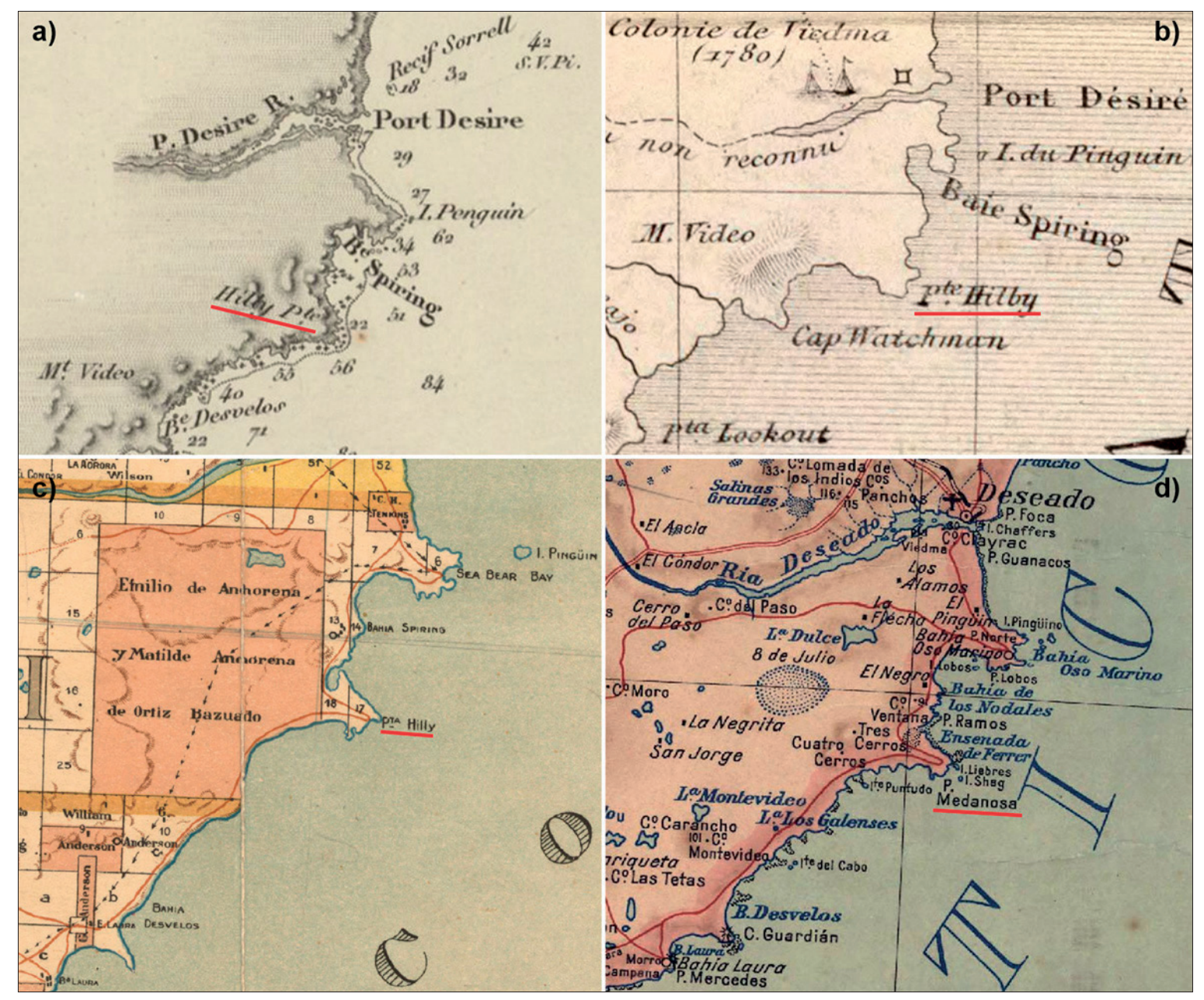

FIGURA 3. EJEMPLOS DE CARTOGRAFÍAS ANTIGUAS EN LOS QUE SE OBSERVA EL CAMBIO EN LA DENOMINACIÓN DE PUNTA MEDANOSA: REFERENCIAS: A) MAPA DE 1853, CARTE DES CÔTES DE LA PATAGONIE ET DES MERS DU CAP HORN (HTTPS://GALLICA.BNF.FR/ARK:/12148/BTVIB53136143V); B) MAPA DE 1865, CARTE DE LA PATAGONIE ET DES ARCHIPELS DE AL TERRE DE FEU DEL MALOUINES ET DES CÔTES OCCIDENTALES (HTTPS://GALLICA.BNF.FR/ ARK:/12148/BTVIB53136143V); MAPA DE 1926 DEL TERRITORIO DE SANTA CRUZ CONFECCIONADO N. M. HERRERA (HTTPS://WWW.IGN.GOB.AR/CARTOGRAFIA-HISTORICA/); Y D) MAPA DE 1945 ELABORADO POR EL INSTITUTO GEOGRÁFICO MILITAR (HTTPS://WWW.IGN.GOB.AR/CARTOGRAFIA-HISTORICA/)

forma ininterrumpida la ganadería ovina para la producción de lanas. En la década de 1930 se intentó explotar comercialmente a los pingüinos de Magallanes para la industria del calzado y la fabricación de carteras, para la cual se llegaron a sacrificar hasta Io mil individuos en una temporada. Sin embargo, este emprendimiento fracasó al poco tiempo (Birabén y Hylton Scott de Birabén I939). En el año I949, el Servicio de Hidrografía Naval construyó un faro cuadrangular de hormigón armado para el auxilio de las embarcaciones en una zona rodeada de islotes (Figura 2.f). A fines del siglo pasado y comienzos del presente se explotó la recolección de macroalgas con fines comerciales. Por último, desde el año 2012 forma parte del Parque Interjurisdiccional Marino Isla Pingüino, reserva creada mediante un convenio entre la Administración de Parques Nacionales y la Provincia de Santa Cruz. Entre los objetivos de este se halla proteger el patrimonio paisajístico, natural y cultural 
de este espacio marítimo y costero, así como promover actividades sostenibles compatibles con la conservación del área³.

\subsection{PRIMEROS ANTECEDENTES ARQUEOLÓGICOS}

Las primeras menciones al registro arqueológico de Punta Medanosa datan de la década de 1930, en las cuales ya se resalta la alta densidad de materiales dejados por las poblaciones cazadoras recolectoras del pasado, la presencia de diversos restos artefactuales (líticos, cerámicas, instrumentos en hueso) y la gran cantidad de entierros de tipo chenque 4 (Aparicio I933-I935; Birabén y Hylton Scott de Birabén I936; Zilio et al. 20I8). Las descripciones brindadas son elocuentes en cuanto a la cantidad de restos arqueológicos existentes, por ejemplo, F. de Aparicio comenta:

recorrimos durante algunos minutos estos amontonamientos y pudimos comprobar que se trata de verdaderos 'conchales' (...) uno de ellos, es, sin embargo, de extensión considerable y, a estar a la cantidad de material que pudimos reunir en el escaso tiempo de nuestra búsqueda, de una riqueza arqueológica extraordinaria (1933-1935: 89).

A fines de la década de I940, esta localidad sería visitada por los arqueólogos europeos Osvaldo Menghin y Marcelo Bormida, quienes afirman que «toda la costa de esta bahía se halla cubierta, casi sin solución de continuidad, por una enorme cantidad de concheros de Mytilys» (s/f: I4) y que «el número de artefactos que se recogen en los concheros y especialmente en sus alrededores es simplemente fabuloso» (s/f: I5). Mientras que, poco después, el arqueólogo aficionado padre Manuel Molina (1976) aporta algunas descripciones de piezas recolectadas durante varias visitas que realiza a esta localidad entre las décadas de I950 y 19770. Entre éstas, se destacan algunas puntas de arpón de hueso (Molina 1967-I970) de características única en el registro de la Patagonia continental, siendo similares a piezas de los canales del extremo austral americano (Zubimendi y Beretta 20I5).

\subsection{LOS ESTUDIOS SISTEMÁTICOS DENTRO DEL PROYECTO ARQUEOLOGÍA DE LA COSTA NORTE DE SANTA CRUZ}

Los estudios arqueológicos sistemáticos en esta localidad se iniciaron a fines de la década de I980 y continúan hasta el presente, aunque en el tiempo han variado

3. Tratado de Creación del «Parque Interjurisdiccional Marino Isla Pingüino» suscripto entre el Estado Nacional y la Provincia de Santa Cruz (http://www.infoleg.gob.ar/infoleglnternet/anexos/205000-209999/206026/norma.htm)

4. El término chenque se utiliza en la región patagónica para referenciar a entierros humanos que se hallan demarcados superficialmente por una cubierta de rocas. Suelen ser estructuras aisladas, ubicadas en posiciones elevadas del terreno (Berón et al. 2000; Goñi y Barrientos 2000; Reyes y Méndez 2010). Lo llamativo de este tipo de practica de entierro es su homogeneidad en toda la Patagonia entre los 1500 años AP y tiempos históricos (Reyes y Méndez 2010; Zilio 2013). Por su parte, en nuestra área de estudio, los chenques han sido datados en un rango cronológico de entre 1000 y 300 años AP (Zilio 2015; Zubimendi et al. 2015). 
según los objetivos de los miembros del equipo, así como también por los vaivenes y avatares de la situación económica y política en ciencia en el país en las últimas décadas, que han influido de diversas formas en la continuidad de las investigaciones.

Los primeros trabajos de campo fueron llevados a cabo por el Dr. E. Moreno, quien realizó algunos sondeos exploratorios y descripciones de sitios (Castro y Moreno I988; Moreno I989-I99I), así como el rescate de un entierro saqueado cerca de La Baliza (Moreno y Romagnino I99I). En el año I999 se volvieron a realizar estudios en esta localidad, en dicha oportunidad se relevó de forma intensiva unos I0.000 $\mathrm{m}^{2}$, al oeste de Punta Medanosa donde se identificaron diez sitios concheros, aunque no se registró su localización (Castro et al. 2003). En los años 2000 y 200 I se realizaron campañas arqueológicas destinadas exclusivamente al estudio de esta localidad, desarrollándose diversas actividades y estudios, que incluyeron relevamientos intensivos y exhaustivos de sitios concheros, entierros de tipo chenque, sondeos exploratorios, estudios tafonómicos sobre restos de aves y recolecciones de materiales líticos y restos óseos hallados en superficie (Castro y Moreno 200o; Castro et al. 200I; Cruz 2004, 2007; Moreno 2009). Los estudios sistemáticos fueron retomados nuevamente en los años 20II y 20I2, cuando se iniciaron una nueva serie de actividades, centradas principalmente en complementar los relevamientos de entierros de tipo chenque, sumado al registro de restos óseos humanos dispersos (Zilio 2015, 2016, 2017, 2019); el sondeo y estudios de la distribución espacial y características geomorfológicas de sitios concheros (Hammond 2013, 2015, 20I7; Hammond et al. 2013, 20I6; Hammond y Zubimendi 2013); así como el relevamiento y muestreo de artefactos de molienda (Ciampagna 20I5). Por último, entre los años 2016 y 2018 los estudios estuvieron centrados principalmente en caracterizar y registrar las alteraciones antrópicas recientes que se evidencian en Punta Medanosa en los últimos años, principalmente en la isla Liebres, sobre los entierros de tipo chenque (Zubimendi 20I9b, 2020), así como al registro de restos históricos y las construcciones -precarias- existentes en toda la localidad.

En resumen, a partir de los antecedentes generados se puede afirmar que Punta Medanosa constituye un espacio litoral con una importante y variada riqueza arqueológica, con características particulares que la destacan entre las evidencias dejadas por las poblaciones cazadoras recolectoras patagónicas del pasado (Castro et al. 200I; Castro et al. 2004; Castro et al. 20II; Hammond et al. 2016; Zangrando 20I8; Zubimendi 20I9a). En esta localidad existen grandes médanos con extensos sitios concheros a lo largo de más siete kilómetros de la costa, lo que denota que ha sido utilizada de forma significativa y redundante (Zubimendi et al. 2005, 2015; Hammond 2015, Zubimendi 2019a). A su vez, la información radiocarbónica muestra que ha sido ocupada al menos desde hace 6300 años AP, lo que denota una secuencia de ocupación de larga duración que abarca parte el Holoceno medio y todo el tardío (Zubimendi et al. 2015; Zubimendi 2019a). Sumado a esto, a lo largo del siglo XX y comienzos del XXI se han recuperado diversos instrumentos artefactuales, tanto a partir de recolecciones realizadas por particulares ${ }^{5}$ como en el marco de

5. Entre los años 2016 y 2018 se relevó la colección particular de la familia Vidal, dueños de la estancia donde 
estudios académicos. Estos constituyen en algunos casos, instrumentos atípicos en la arqueología patagónica y permiten conocer aún más la variabilidad de las formas de uso y explotación de los recursos por parte de las poblaciones cazadoras recolectoras patagónicas. Entre ellos podemos mencionar gran cantidad de puntas de arpón de hueso (Zubimendi y Beretta 2015), punzones de madera (Ciampagna 20I8), retocadores y agujas de hueso, entre otros.

Punta Medanosa también se destaca por la gran cantidad de estructuras de piedra que presumiblemente corresponden a entierros humanos, en su inmensa mayoría de tipo chenque (Castro y Moreno 2000; Castro et al. 2002; Zilio et al. 2013), aunque también se han registrado otros tipos de entierros (Zilio 2015, 20I7, 20I9). En el caso de los chenques, se ha planteado en base a su gran concentración en ciertas zonas de esta localidad, que algunos espacios habrían sido utilizados como lugares persistentes para el entierro de restos humanos hace aproximadamente 700 años AP (Zilio 2015; Zubimendi et al. 2015). Los restantes tipos de entierros tienen cronologías que abarcan desde los 5000 hasta 1500 años AP (Zilio 2015; Zubimendi 2019a).

\section{METODOLOGÍA}

A partir de los datos obtenidos desde los inicios de los estudios sistemáticos en Punta Medanosa, decidimos volcar toda la información disponible de arqueología prehistórica (sitios concheros, entierros, etc.), histórica (pecios, restos de naufragios) y contextual (puntos de control, del paisaje o construcciones) en un SIG. De esta forma, pretendemos generar un entorno georreferenciado que permita obtener una imagen global de esta localidad y confeccionar diferentes cartografías temáticas de interés para el proyecto marco, así como delinear futuros análisis espaciales y políticas de gestión y conservación.

\subsection{DESCRIPCIÓN DE LAS CAPAS DE DATOS CONTEXTUALES}

Para la confección de la base de datos utilizando SIG se emplearon imágenes satelitales de alta resolución. Entre los datos vectoriales se usaron las coberturas de los departamentos y límites político-administrativos del SIG250 del Instituto Geográfico Nacional (IGN) a escala I:250.000, que tienen proyección Gauss Krüger Argentina faja 2 y datum WGS84. Los datos espaciales se manejaron con software específico (ArcMap Io.2 y QGIS). Al mismo tiempo que los puntos registrados fueron guardados en los posicionadores satelitales, se anotaron en libretas de campo, y en caso de recolecciones de materiales, en los rótulos correspondientes, de esta forma se obtuvieron varios controles cruzados de los puntos registrados. Por otro lado, a

se halla Punta Medanosa. Esto nos permitió identificar unas 3500 piezas que se hallan en su poder, entre ellos gran cantidad de instrumentos líticos, como puntas de proyectil, bifaces, agujas, puntas destacadas, raspadores; instrumentos en hueso, entre los que destacan puntas de arpón y retocadores; así como también agujas de madera. 
partir de las imágenes satelitales y la contrastación en el campo se generó una capa de caminos (rutas y huellas de vehículos). En todos los casos se decidió emplear el sistema de coordenadas UTM y el datum WGS84.
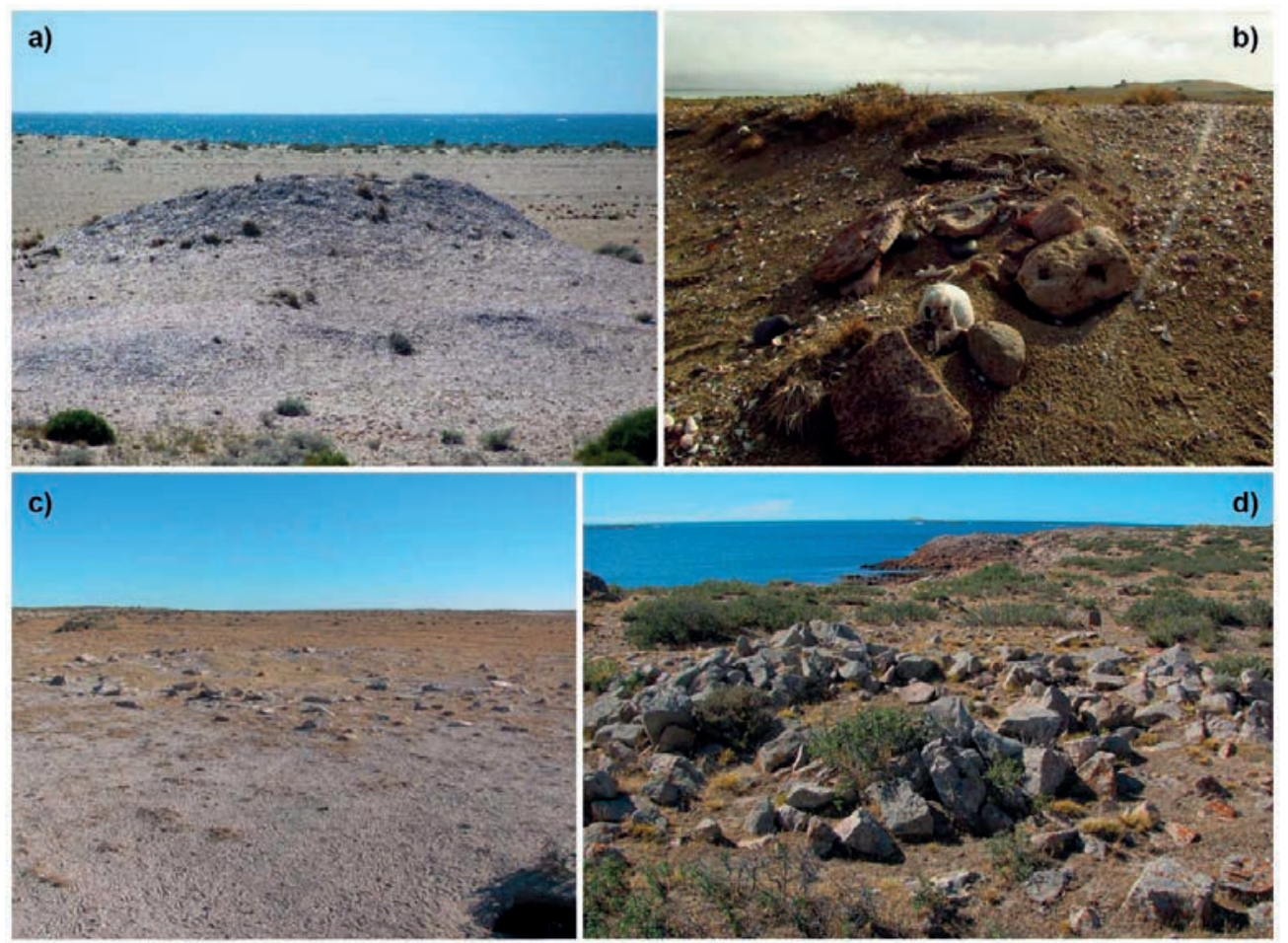

d)
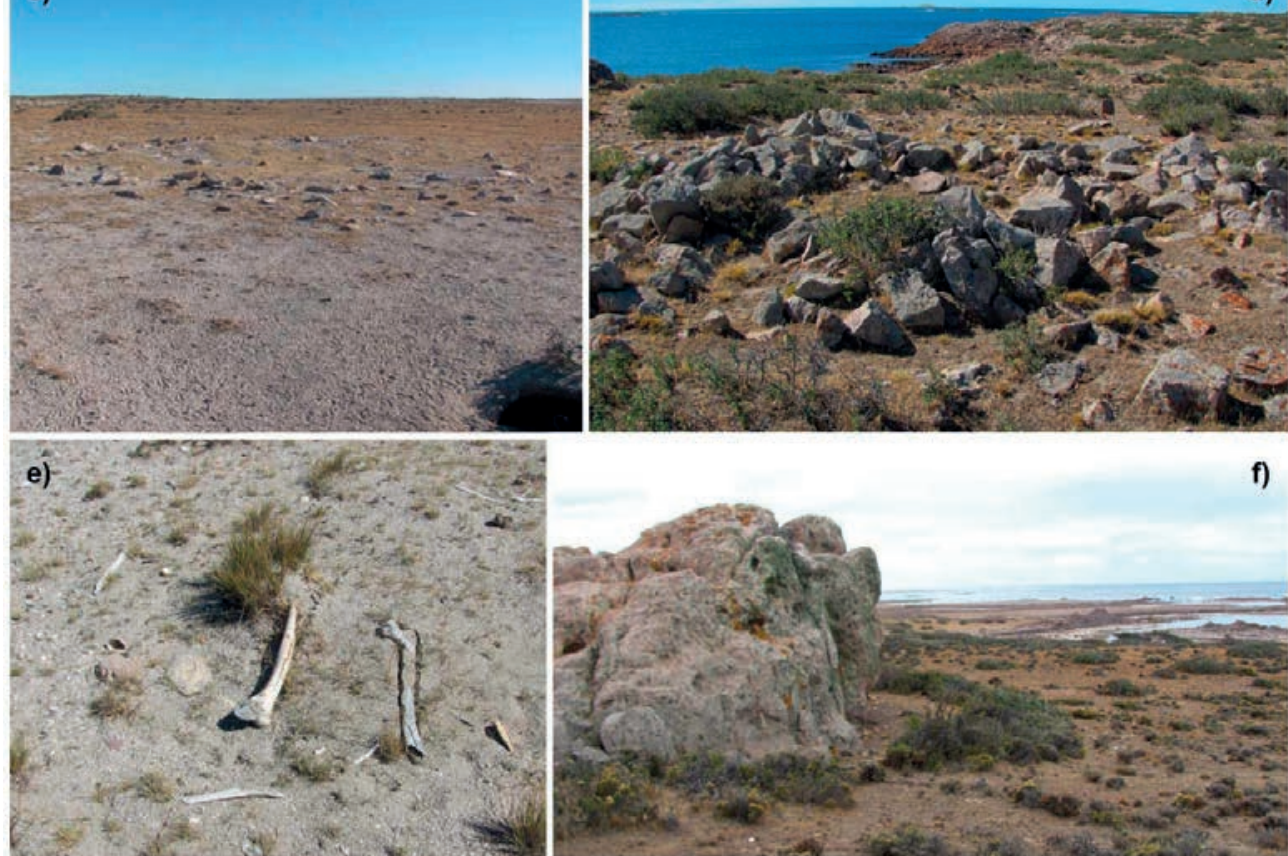

FIGURA 4. EJEMPLOS DE SITIOS ARQUEOLÓGICOS PREHISTÓRICOS IDENTIFICADOS EN PUNTA MEDANOSA. REFERENCIAS: A) CONCHERO EN MÉDANO EN PUNTA BUQUE; B) ENTIERRO EN MÉDANO EN EROSIÓN EN PLAYA CENTRAL; C) ENTIERRO EN ANILLO EN PUNTA BUQUE; D) ENTIERRO TIPO CHENQUE EN BALIZA; E) HUESOS HUMANOS EN SUPERFICIE EN PLAYA CENTRAL; F) ABRIGO ROCOSO EN COSTA SUDESTE. LAS UBICACIONES DE LAS FOTOS DE EJEMPLO SE HALLAN EN LA FIGURA 8. Figura elaboración propia

De acuerdo con la información contextual asociada a cada punto registrado en las libretas de campo y fichas descriptivas, se los clasificó como:

I. concheros (Figura 4.a);

2. entierros, dentro de estos se agrupan las modalidades en médano (Figura 4.b), en anillo (Figura 4.c) y chenques (Figura 4.d) (ver Zilio 2015);

3. huesos humanos dispersos, probablemente producto de alteraciones naturales o antrópicas (Figura 4.e);

4. abrigos rocosos (Figura 4.f); 
5. restos históricos, como pecios (Figura 5.a) y partes de naufragios (Figura 5.b);

6. estructuras recientes, entre estos pircados (Figura 5.c), fogones (Figura 5.d) y basureros (Figura 5.e);

7. construcciones precarias (Figura 5.f), hechas por los dueños de la estancia; y

8. otros, que abarca a los puntos destacados del paisaje, de control o de transectas (por ejemplo, al inicio y al final).
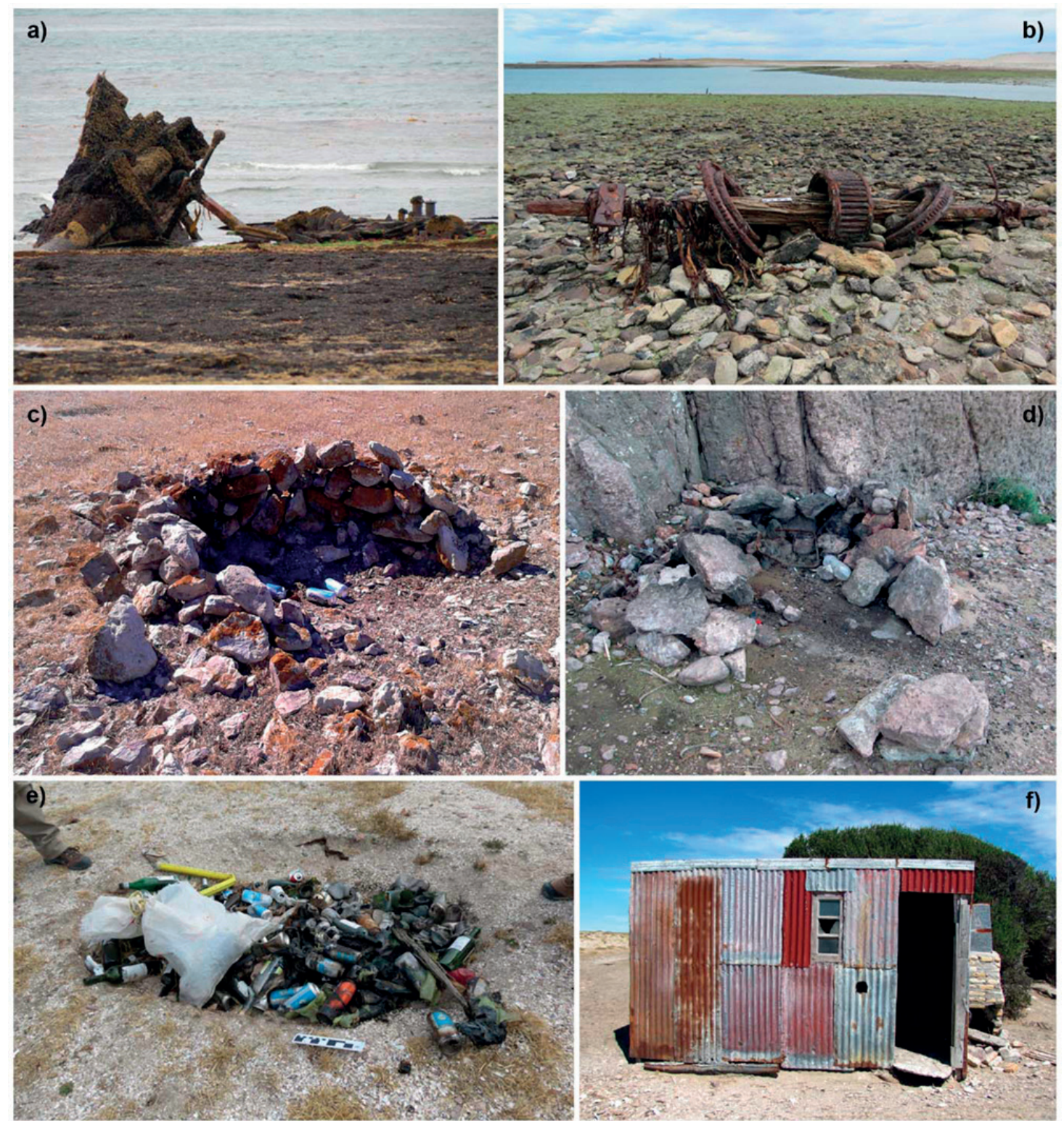

FIGURA 5. EIEMPLO DE RESTOS ARQUEOLÓGICOS HISTÓRICOS Y ESTRUCTURAS RECIENTES IDENTIFICADAS EN PUNTA MEDANOSA. REFERENCIAS: A) PECIO EN EL EXTREMO INFERIOR DEL INTERMAREAL AL NORTE DE ISLA LIBRES; B) MOLINETE DE BARCO EN EL INTERMAREAL, AL NOROESTE DE ISLA LIEBRES; C) PIRCADO CONSTRUIDO EN ISLA LIEBRES (CON BASURA EN SU INTERIOR); D) FOGÓN CONSTRUIDO CONTRA BLOQUE ROCOSO EN BALIZA; E) POZO DE BASURA COLMATADO EN ISLA LIEBRES; F) PUESTO DE ALGUEROS CONSTRUIDO EN PUNTA BUQUE. LAS UBICACIONES DE LAS FOTOS DE EJEMPLO SE HALLAN EN LA FIGURA 8. Figura elaboración propia 


\subsection{FUENTES DE DATOS ARQUEOLÓGICOS GEORREFERENCIADAS}

Sin duda, existe una importante diferencia en la información que se ha empleado para crear la base de datos de Punta Medanosa, ya que varió según el año de captura -que abarca un rango de 30 años-, el posicionador satelital, las personas que registraron los puntos, el error estimado, así como también -y probablemente más importante aún- el objetivo de investigación y las metodologías empleadas en cada caso (Tabla I). Es interesante resaltar que las diferentes actividades que se desarrollaron en los trabajos de campo permitieron observar que los datos obtenidos, a pesar de que han variado los objetivos, presentan un énfasis en los estudios de distribución de sitios, tanto concheros como chenques, y se ha abarcado espacialmente casi todas las zonas de Punta Medanosa, aunque algunas han sido relevadas con mayor intensidad que otras.

\begin{tabular}{|c|c|c|c|c|c|c|c|c|c|}
\hline \multirow[b]{2}{*}{ CAMPAÑA } & \multirow{2}{*}{$\begin{array}{c}\text { FORMA } \\
\text { DE } \\
\text { REGISTRO }\end{array}$} & \multirow{2}{*}{\begin{tabular}{|c|} 
ERROR \\
ESTIMADO \\
\end{tabular}} & \multirow[b]{2}{*}{ COLECTORES } & \multicolumn{3}{|c|}{ ESTUDIOS DISTRIBUCIONALES } & \multirow[b]{2}{*}{ EXCAVACIONES } & \multirow{2}{*}{ RECOLECCIONES } & \multirow{2}{*}{$\begin{array}{c}\text { CANTIDAD } \\
\text { DE } \\
\text { PUNTOS }\end{array}$} \\
\hline & & & & CONCHEROS & ENTIERROS & $\begin{array}{c}\text { ESTRUCTURAS } \\
\text { RECIENTES }\end{array}$ & & & \\
\hline $1987-1991$ & $\begin{array}{l}\text { Ubicación } \\
\text { relativa }\end{array}$ & $\sim 100 \mathrm{~m}$ & E. Moreno & $x$ & - & - & $x$ & - & 6 \\
\hline 1999 & $\begin{array}{l}\text { Magellan } \\
\text { Lowrance } \\
\text { GM-100 }\end{array}$ & $\begin{array}{c}15-100 \mathrm{~m} \\
(\mathrm{~S} / \mathrm{A})\end{array}$ & A. Castro & $x$ & - & - & - & - & 2 \\
\hline 2000 & $\begin{array}{l}\text { Garmin } \\
\text { Etrex }\end{array}$ & $\begin{array}{c}15-100 \mathrm{~m} \\
(\mathrm{~S} / \mathrm{A})\end{array}$ & $\begin{array}{l}\text { A. Castro, E. } \\
\text { Moreno }\end{array}$ & $x$ & - & - & $x$ & $x$ & 143 \\
\hline 2001 & $\begin{array}{l}\text { Garmin } \\
\text { Etrex }\end{array}$ & $10 \mathrm{~m}$ & $\begin{array}{l}\text { A. Castro y E. } \\
\text { Moreno y C. } \\
\text { Peña }\end{array}$ & - & $x$ & - & $x$ & $x$ & 222 \\
\hline 2011 & $\begin{array}{l}\text { Garmin } \\
\text { Etrex, } \\
\text { Legend y } \\
\text { Vista }\end{array}$ & $4-6 m$ & $\begin{array}{l}\text { L. Ciampagna, } \\
\text { H. Hammond, } \\
\text { L. Zilio y M. } \\
\text { Zubimendi }\end{array}$ & - & $x$ & - & $x$ & $x$ & 56 \\
\hline 2012 & $\begin{array}{l}\text { Garmin } \\
\text { Etrex, } \\
\text { Legend y } \\
\text { Vista }\end{array}$ & $4-6 \mathrm{~m}$ & $\begin{array}{l}\text { H. Hammond, } \\
\text { L. Zilio y M. } \\
\text { Zubimendi }\end{array}$ & $x$ & $x$ & - & $x$ & $x$ & 169 \\
\hline 2016 & $\begin{array}{l}\text { Garmin } \\
\text { Legend }\end{array}$ & $4-6 m$ & M. Zubimendi & $x$ & $x$ & $x$ & - & - & 42 \\
\hline 2017 & $\begin{array}{l}\text { Garmin } \\
\text { Etrex } 30 x\end{array}$ & $4-6 m$ & M. Zubimendi & - & $\mathrm{x}$ & $x$ & $x$ & $x$ & 59 \\
\hline 2018 & $\begin{array}{l}\text { Garmin } \\
\text { Etrex } 30 x\end{array}$ & $4-6 m$ & M. Zubimendi & - & $x$ & $x$ & - & - & 69 \\
\hline
\end{tabular}

TABLA 1. METADATOS DE LAS FUENTES DE DATOS ARQUEOLÓGICOS GEORREFERENCIADOS Y OBJETIVOS EN CADA CAMPAÑA 
En las últimas décadas del siglo XX se realizaron tres viajes de campaña dirigidos por el Dr. Moreno -entonces estudiante- en las que se realizaron diversas actividades que quedaron plasmadas en informes técnicos inéditos (Castro y Moreno I988; Moreno I989-I99I; Moreno y Romagnino I99I). A partir de la descripción de la ubicación de cinco sondeos estratigráficos y un rescate arqueológico de un entierro de tipo chenque saqueado, es posible localizarlos con cierta precisión, con un error estimado de ca. Ioo $\mathrm{m}$. En un caso, la ubicación es más precisa dado que corresponde a un alero rocoso aislado en la costa este y fácilmente localizable (Figura 4.f).

La primera oportunidad en la que se empleó un posicionador satelital fue en febrero de 1999 cuando se relevaron diez concheros en un área de Ioo x I000 m con el lado mayor paralelo a la línea de costa (Castro et al. 200I, 2003), aunque sólo se registraron los extremos hacia el mar del área relevada. En la campaña arqueológica del año 2000 se incluyó dentro de su diseño el empleo masivo de información geográfica mediante un posicionador. Durante estos trabajos de campo se relevaron de forma intensiva concheros en toda la franja norte, compuesta principalmente por mantos de dunas, así como la zona interior o central de Punta Medanosa. A su vez, se registraron algunas estructuras de entierro, sobre todo en la zona de la baliza y la costa inmediatamente al sur. Conviene tener en cuenta que los datos registrados antes de mayo de 2000 contaban con el denominado error de S/A (disponibilidad selectiva por sus siglas en ingles), que era una degradación intencional y aleatoria de la señal GPS, ya que, al ser esta de origen militar, se pretendía evitar la excesiva precisión de los GPS comerciales. Es por ello que los puntos registrados hasta ese momento cuentan con un rango de error horizontal estimado entre 15 y $100 \mathrm{~m}$.

En el año 200I se emplearon dos posicionados satelitales y los estudios se focalizaron especialmente en lograr un relevamiento exhaustivo de estructuras de entierro de tipo chenque a lo largo de casi todo el perímetro costero. Esta actividad estuvo dirigida por la estudiante Catalina Peña, y participaron también otros integrantes del equipo de investigación. Sólo quedó sin relevar el tramo de costa acantilada del sudoeste. Cada chenque fue posicionado y descripto según una ficha especialmente diseñada (Castro et al. 2002). También se tomó la posición geográfica de algunos concheros, aunque con menor intensidad que en la campaña anterior.

En los años 2004, 2006 y 2007 se realizaron visitas esporádicas y de corta duración, sin que se registren datos geográficos. Recién en el 20II se reinician los estudios sistemáticos, empleándose tres posicionadores satelitales. En dicha oportunidad, las investigaciones estuvieron dirigidos a la realización de las tesis doctorales de, en ese entonces, las lic. L. Ciampagna, H. Hammond y el lic. L. Zilio. En este sentido, se realizó un muestreo sistemático de morteros; un relevamiento preliminar de las características y el sondeo de sitios concheros en la franja norte; y el registro de chenques al norte de la baliza, respectivamente. Al año siguiente, los objetivos fueron realizar estudios de distribución de sitios concheros mediante transectas lineales en las franjas norte y este, a cargo de H. Hammond; y relevar entierros de tipo chenque en la franja este y sudeste, dirigidos por L. Zilio. Para 
ambos estudios se confeccionaron fichas descriptivas en las que se consignaba la posición geográfica, dato que luego fue utilizado para realizar diversos análisis.

En el año 2016 los relevamientos, dirigidos por el Dr. M. Zubimendi, se concentraron en la isla Liebres, ubicada en el extremo este y, al oeste, en torno a la ensenada Ferrer. En la primera realizó un relevamiento exhaustivo de las estructuras de entierro de tipo chenque que existen en esta isla debido a referencias de intensas alteraciones que se producían por parte de visitantes ocasionales (ver Zubimendi 2019b, 2020). En tanto que cerca de ensenada Ferrer realizó un estudio de distribución de sitios mediante transectas. Se destaca que esta fue la primera campaña en la que se registraron estructuras de piedra recientes, fogones o pircados, que en algunos casos han afectado a chenques cercanos.

En la campaña del año 20I7, los trabajos se orientaron a continuar el relevamiento sistemático y continuado de las alteraciones antrópicas recientes, sobre todo en la isla Liebres, incluyendo algunas nuevas construidas entre los años 2016 y 20I7. También se relevaron algunas estructuras recientes cerca de La Baliza. Por último, se sondearon algunos sitios concheros en la franja norte. $\mathrm{Al}$ año siguiente, último del que se cuenta con datos georeferenciados, se realizó un nuevo relevamiento de las alteraciones recientes y sitios arqueológicos en isla Liebres. En esta oportunidad se trabajó junto con la Lic. Soledad Caracotche y la Dra. Rocío Blanco, de la Administración de Parques Nacionales, dado que dicha isla pertenece al Parque interjurisdiccional marino Isla Pingüino. Se registró por primera vez la posición geográfica de restos históricos, como la presencia de un pecio en el intermareal y diversos restos de naufragios tanto en el intermareal, la línea de marea y en el interior de la isla Liebres, incluso algunos de ellos formado parte de estructuras recientes.

\section{RESULTADOS}

En la Tabla 2 se presenta la superficie relevada durante cada campaña arqueológica, y los puntos generados, especificando el número de puntos repetidos y tipos de puntos según la clasificación general mencionada previamente. En la Figura 6.a se muestra la evolución cuantitativa en la cantidad de datos a lo largo de los años, con aumentos mayores en aquellas campañas con objetivos de trabajo -relacionada con las investigaciones particulares llevadas a cabo por los miembros del equipo de investigación- orientadas a relevamientos sistemáticos e intensivos de sitios: concheros en los años 2000 y 20I2, entierros en el 200I, y estructuras recientes desde el 20i6 (Figura 6.b). Por medio de la superposición de los datos se constata que el crecimiento en la cantidad de registros es acompañado por la de puntos repetidos (Tabla 2). En relación con estos últimos, para poder corroborar los mismos, se tuvo en cuenta el error estimado de los GPS, así como también la información volcada en las libretas de campo, ya que en algunos casos se registró un contexto -conchero, entierro o alteración reciente, por ejemplo- en más de una oportunidad. En las últimas campañas, se registran altos porcentajes de puntos repetidos, aunque conviene destacar que se realizó un estudio longitudinal de los chenques de isla Liebres, lo que explicaría parte de esta repetición. En este 
sentido, se volvió a ubicar algunos sitios y se está realizando una evaluación continuada de los cambios que sufren por causas antrópicas, mientras que para las estructuras recientes se analizan sus cambios y evolución. Por otro lado, a lo largo de las sucesivas campañas se ha registrado una mayor diversidad de tipos de puntos, destacándose en los últimos años la categoría estructuras recientes, producidas por personas -turistas ocasionales y pescadores principalmente-que visitan la localidad arqueológica y generan pircados o fogones, en algunos casos modificando chenques preexistentes (Zubimendi 20I9b, 2020), así como también restos de naufragios registrados en el último relevamiento. Por su parte, los puntos en la categoría otros presentan aumentos importantes en su frecuencia en aquellas campañas en las que se realizaron transectas de muestreo sistemáticas (2000, 200I y 2012), dado que mayormente corresponden a puntos de control.

\begin{tabular}{|c|c|c|c|c|c|c|c|c|c|c|}
\hline \multirow{2}{*}{ CAPA } & \multirow{2}{*}{$\begin{array}{l}\text { SUPERFICIE } \\
\text { RELEVADA } \\
\text { (APROX.) }\end{array}$} & \multirow{2}{*}{$\begin{array}{c}\text { PUNTOS } \\
\text { (N Y \% } \\
\text { REPETIDOS) }\end{array}$} & \multicolumn{8}{|c|}{ TIPOS } \\
\hline & & & ABRIGOS & CONCHEROS & ENTIERROS & $\begin{array}{c}\text { ESTRUCTURAS } \\
\text { RECIENTES }\end{array}$ & $\begin{array}{c}\text { HUESOS } \\
\text { HUMANOS }\end{array}$ & NAUFRAGIOS & PUESTOS & OTROS \\
\hline $\begin{array}{l}1987- \\
1991\end{array}$ & $s / d$ & 6 & $1(16,7 \%)$ & $4(66,8 \%)$ & $1(16,7 \%)$ & - & - & - & - & - \\
\hline 1999 & $0,1 \mathrm{~km}^{2}$ & 2 & - & - & - & - & - & - & - & $2(100 \%)$ \\
\hline 2000 & $20 \mathrm{~km}^{2}$ & $\begin{array}{l}143(1 ; \\
0,6 \%)\end{array}$ & - & $68(47,6 \%)$ & $56(39,2 \%)$ & - & - & - & - & $\begin{array}{c}19 \\
(13,3 \%)\end{array}$ \\
\hline 2001 & $6 \mathrm{~km}^{2}$ & $\begin{array}{c}222(38 ; \\
17,1 \%)\end{array}$ & - & $21(9,5 \%)$ & $150(67,6 \%)$ & $1(0,5 \%)$ & $11(5 \%)$ & - & - & $\begin{array}{c}39 \\
(17,6 \%) \\
\end{array}$ \\
\hline 2011 & $3,5 \mathrm{~km}^{2}$ & $\begin{array}{l}56(24 ; \\
42,8 \%)\end{array}$ & - & $33(58,6 \%)$ & $19(33,9 \%)$ & - & $2(3,6 \%)$ & - & $1(1,8 \%)$ & $1(1,8 \%)$ \\
\hline 2012 & $7 \mathrm{~km}^{2}$ & $\begin{array}{c}169(39 ; \\
23 \%)\end{array}$ & $2(1,2 \%)$ & $99(58,6 \%)$ & $30(17,8 \%)$ & $1(0,6 \%)$ & $11(6,5 \%)$ & - & - & $\begin{array}{c}26 \\
(15,4 \%) \\
\end{array}$ \\
\hline 2016 & $1,5 \mathrm{~km}^{2}$ & $\begin{array}{l}42(10 ; \\
23,8 \%)\end{array}$ & - & $24(57,1 \%)$ & $7(16,7 \%)$ & $6(14,3 \%)$ & $2(4,8 \%)$ & - & $1(2,4 \%)$ & $2(4,8 \%)$ \\
\hline 2017 & $3,5 \mathrm{~km}^{2}$ & $\begin{array}{l}59(20 \\
33,9 \%)\end{array}$ & - & $12(20,3 \%)$ & $7(11,9 \%)$ & $17(40,7 \%)$ & $6(10,2 \%)$ & $1(1,7 \%)$ & $1(1,7 \%)$ & $8(13,6 \%)$ \\
\hline 2018 & $0,4 \mathrm{~km}^{2}$ & $\begin{array}{l}69(21 ; \\
30,4 \%)\end{array}$ & - & $2(2,9 \%)$ & $28(40,6 \%)$ & $27(39,1 \%)$ & - & $12(17,4 \%)$ & - & - \\
\hline Total & - & $\begin{array}{c}768 \\
(153 ; 19,9 \%)\end{array}$ & $3(0,4 \%)$ & $263(34,2 \%)$ & $298(38,8 \%)$ & $59(7,7 \%)$ & $32(4,2 \%)$ & $13(1,7 \%)$ & $\begin{array}{c}3 \\
(0,4 \%)\end{array}$ & $\begin{array}{c}97 \\
(12,6 \%)\end{array}$ \\
\hline
\end{tabular}

TABLA 2. CANTIDAD Y TIPOS DE PUNTOS REGISTRADOS EN CADA CAMPAÑA DE TRABAJOS DE CAMPO

En relación con el conocimiento arqueológico de Punta Medanosa, la base de datos creada permite referenciar y observar cómo fue la construcción de la información espacial generada en esta localidad por parte del equipo de investigación a lo largo de 30 años. Como se comprueba en los datos surgidos de las diferentes campañas (Figura 7), a pesar de los diversos objetivos a lo largo de los años, se observa un énfasis en la distribución de sitios, tanto concheros como chenques, y en una amplia cobertura y superficie relevada, ya que se realizaron relevamientos en casi toda Punta Medanosa, aunque la superficie relevada en cada campaña varía entre 0 , I y $20 \mathrm{~km}^{2}$ (Tabla 2). 
a)

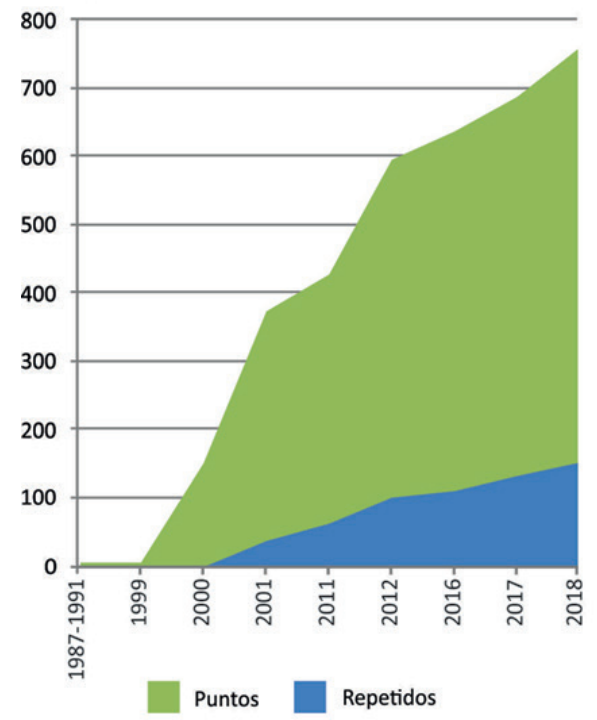

b)

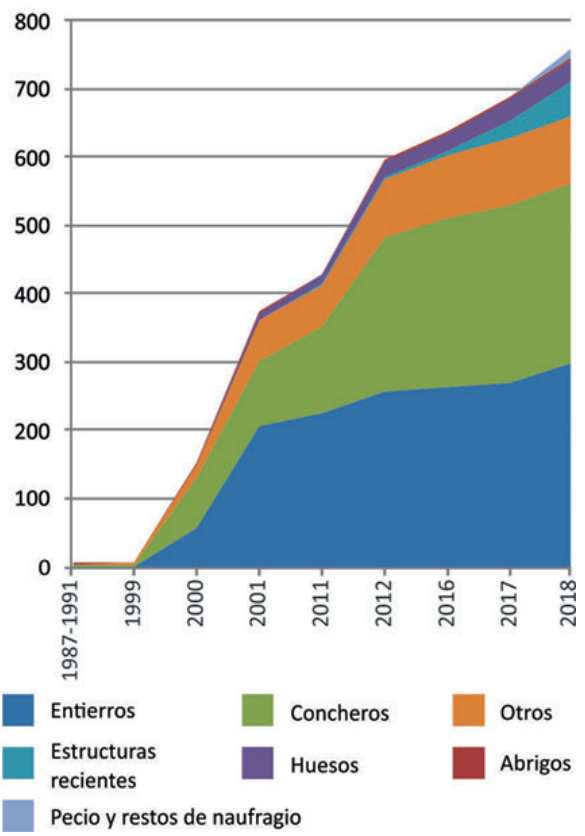

FIGURA 6. A) GRÁFICO DE FRECUENCIAS ACUMULADAS DE LOS PUNTOS REGISTRADOS, INCLUYENDO AQUELLOS REPETIDOS; B) GRÁFICO DE FRECUENCIAS ACUMULADAS SEGÚN TIPOS DE PUNTOS REGISTRADOS. Figura elaboración propia

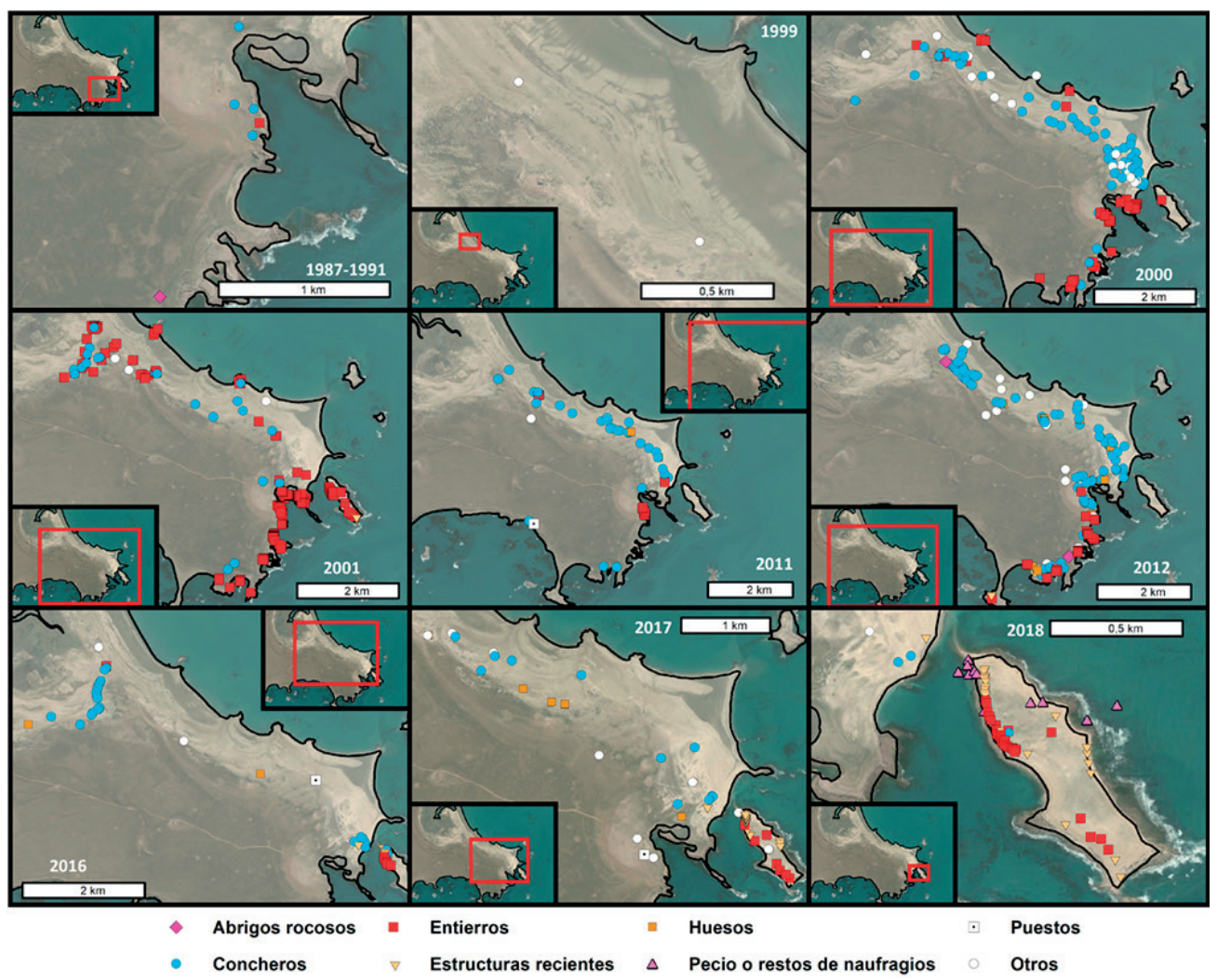

FIGURA 7. UBICACIÓN Y TIPOS DE PUNTOS REGISTRAdOS EN CADA CAMPAÑA DE TRABAJOS DE CAMPO. Figura elaboración propia 


\section{DISCUSIÓN}

\subsection{LA CARTA ARQUEOLÓGICA}

El ordenamiento y sistematización de la información espacial desarrollada en el marco del proyecto Arqueología de la Costa Norte de Santa Cruz ha permitido confeccionar una base de datos geográfica de Punta Medanosa. A partir de esta se construyó una carta arqueológica en la que se resumen las principales características del registro arqueológico de esta localidad ${ }^{6}$, constituyendo una imagen general y abarcativa (Figura 8). En esta carta, siguiendo lo realizado por Magnin (20I7), se excluyeron los puntos de la categoría «otros», ya que no corresponden a entidades arqueológicas o históricas y, por lo tanto, no son elementos de interés para la carta. En el caso de los puntos repetidos se decidió representar sólo los de la fuente más reciente. De esta forma, la carta arqueológica incluye 502 puntos que referencian a entidades arqueológicas prehistóricas e históricas, estructuras recientes y construcciones; así como la traza de rutas y huellas generadas por la circulación de vehículos. La carta puede constituir un producto de gran valor para la gestión por parte de diversas instituciones que deben velar por su protección y salvaguarda (Gallego Muñóz 20I3; Panizza y Oliva 20I8). Sin duda, al poseer toda la información resumida en una única carta arqueológica será posible llamar la atención sobre la necesidad de protección y generación de políticas de gestión cultural para evitar que continúe el acelerado proceso de alteración y destrucción del registro arqueológico en el que se encuentra (Zubimendi 20I9b, 2020).

La carta arqueológica permite presentar, confirmar y ampliar de forma visual algunas tendencias y resultados expresados previamente en diferentes trabajos del equipo de investigación. Por ejemplo, ya desde los primeros relevamientos se había planteado una clara diferenciación entre la costa norte, caracterizada por los extensos bancos de dunas en deflación, y la costa este y sur, mayormente rocosa (Castro et al. 200I). En la primera predominan ampliamente los concheros insertos en una matriz de dunas y en la segunda los entierros de tipo chenque. Sin embargo, podemos comprobar que esta diferenciación no es estricta ya que, en algunos sectores, existen chenques y concheros muy cercanos entre sí. También es posible identificar o sectorizar ${ }^{7}$ nueve zonas dentro de la localidad arqueológica Punta Medanosa, entre las que se observan diferencias y similitudes (Tabla 3). La mayoría cuentan con información sistemática a lo largo de casi todas las campañas. Por su parte, la excepción lo constituyen: la isla Liebres, que fue sólo relevada en cuatro oportunidades -a pesar de tener una interesante concentración de

6. La capa shapefile de puntos de la carta arqueológica se encuentra disponible para su consulta en la web de la revista Espacio, Tiempo y Forma. Serie I: http://revistas.uned.es/index.php/ETFl/index y en el Servicio de Difusión de la Creación Intelectual (SeDiCl), que es el Repositorio Institucional de la Universidad Nacional de La Plata: http://sedici.unlp.edu.ar/handle/10915/122320.

7. Esta sectorización coincide en parte con la planteada en un trabajo previo para el caso de los entierros de tipo chenques a partir del relevamiento realizado en el año 2001 (Zilio et al. 2013). 
chenques y actualmente existir importantes alteraciones modernas que afectan al registro arqueológico prehistórico e histórico (ver Zubimendi 2019b, 2020)-; la ensenada Ferrer, que sólo fue relevada sistemáticamente en tres oportunidades; y por sobre todo la costa sudoeste, para la cual sólo se cuenta con tres puntos que corresponden a una pequeña concentración de tiestos cerámicos, un chenque y un puesto, puntos obtenidos en relevamientos asistemáticos. Por otro lado, la meseta alta fue relevada de forma sistemática mediante transectas en dos oportunidades, y asistemáticamente en varias ocasiones, pero no se han registrado concentraciones de materiales arqueológicos, lo que refleja, sin duda, una densidad artefactual extremadamente baja o nula 8 .

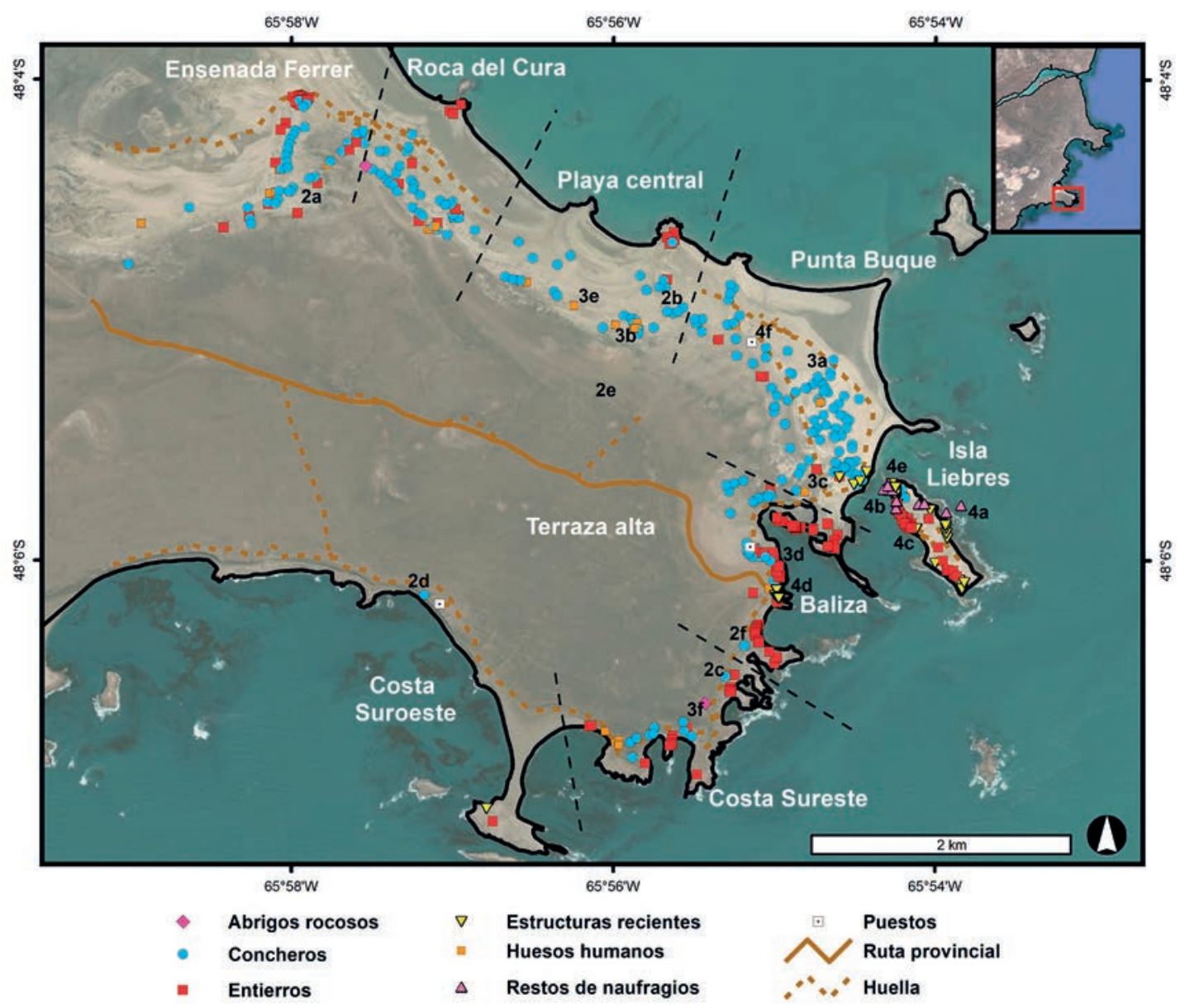

FIGURA 8. CARTA ARQUEOLÓGICA DE PUNTA MEDANOSA. LAS LETRAS Y NÚMEROS EN NEGRO CORRESPONDEN A LA UBICACIÓN DE LAS FOTOS DE LAS FIGURAS 2, 3 Y 4 . Figura elaboración propia

8. En el año 2000 se realizaron siete transectas en sentido norte-sur, en las que se relevó la presencia de sitios, y si bien se observaron algunos hallazgos aislados, estos no fueron posicionados (Castro et al. 2003). Igualmente, la densidad artefactual en la meseta alta fue extremadamente baja, en especial si lo comparamos con las altas densidades observadas en los sitios concheros cercanos. Asimismo, en el año 2012 también se relevó parte de la meseta alta, sin que se registraran hallazgos líticos (Hammond 2015, 2019, Hammond et al. 2013). 


\begin{tabular}{|c|c|c|c|c|c|c|c|c|c|}
\hline SECTORES & $\begin{array}{c}\text { N } \\
\text { RELEVAMIENTOS }\end{array}$ & CONCHEROS & ENTIERROS & $\begin{array}{l}\text { HUESOS } \\
\text { HUMANOS }\end{array}$ & $\begin{array}{l}\text { ABRIGOS } \\
\text { Rocosos }\end{array}$ & $\begin{array}{c}\text { RESTOS } \\
\text { HISTÓRICOS }\end{array}$ & $\begin{array}{l}\text { ESTRUCTURAS } \\
\text { RECIENTES }\end{array}$ & PUESTOS & $\begin{array}{c}\mathbf{N} \\
\text { PUNTOS }\end{array}$ \\
\hline $\begin{array}{l}\text { Ensenada } \\
\text { Ferrer }\end{array}$ & 3 & $35(52,2 \%)$ & $26(38,8 \%)$ & $6(9 \%)$ & - & - & - & - & 67 \\
\hline $\begin{array}{l}\text { Roca del } \\
\text { Cura }\end{array}$ & 5 & $31(63,3 \%)$ & $12(24,5 \%)$ & $5(10,2 \%)$ & $1(2 \%)$ & - & - & - & 49 \\
\hline $\begin{array}{l}\text { Punta } \\
\text { Buque }\end{array}$ & 5 & $92(87,6 \%)$ & $5(4,8 \%)$ & $3(2,9 \%)$ & - & - & $4(3,8 \%)$ & $1(1 \%)$ & 105 \\
\hline Isla Liebres & 4 & $4(4,7 \%)$ & $43(50,6 \%)$ & - & - & $12(14,1 \%)$ & $26(30,6 \%)$ & - & 85 \\
\hline Baliza & 5 & $24(21,8 \%)$ & 77 (70\%) & $1(0,9 \%)$ & - & - & $7(6,4 \%)$ & $1(0,9 \%)$ & 110 \\
\hline $\begin{array}{l}\text { Costa } \\
\text { sudoeste }\end{array}$ & 1 & $1(25 \%)$ & $1(25 \%)$ & - & - & - & $1(25 \%)$ & $1(25 \%)$ & 4 \\
\hline Terraza alta & 2 & - & - & - & - & - & - & - & 0 \\
\hline Total & - & $229(45,4 \%)$ & $191(37,9 \%)$ & $27(5,4 \%)$ & $2(0,4 \%)$ & $12(2,4 \%)$ & $38(7,9 \%)$ & $3(0,6 \%)$ & 502 \\
\hline
\end{tabular}

TABLA 3. SECTORES EN LAS QUE SE PUEDE DIFERENCIAR A PARTIR DE LA CARTA ARQUEOLÓGICA LA LOCALIDAD PUNTA MEDANOSA DE ACUERDO CON LA FRECUENCIA, DENSIDAD Y TIPO DE REGISTRO ARQUEOLÓGICO

\subsection{LOS SITIOS PREHISTÓRICOS}

La presencia de entierros de tipo chenque se concentra principalmente en cercanías de la línea de costa, siguiendo el contorno de esta, en especial en la costa este, pero también en algunos pequeños tómbolos en la costa norte (Zilio et al. 2013). Sin embargo, llama también la atención la concentración de chenques en la zona norte de la ensenada Ferrer y otros pocos que se hallan en contextos de médanos, donde en general se presentan aislados o formando agrupamientos de no más de tres estructuras. Otro resultado interesante de la sumatoria de los relevamientos realizados es la constatación de la presencia de huesos humanos expuestos mayormente en las dunas ubicadas en la parte interna de la playa de cordones litorales con dunas, o sea, aquellas dunas más cercanas a la meseta alta. Uno de estos hallazgos, denominado $\mathrm{PM}_{3}$, corresponde a un entierro humano disturbado que arrojó un fechado de $4.970 \pm$ Ioo años AP (LP-3034), el más antiguo para este tipo de entierros en la costa norte de Santa Cruz (Zilio 20I6). A sólo 25 $\mathrm{m}$ se han registrado dos entierros no alterados de similares características, lo que permite plantear la posibilidad de que tengan una antigüedad similar y que haya constituido un lugar recurrente de entierro en dunas (Zubimendi 2019a). Este tipo de práctica de entierro se desarrolló en el área de estudio en un rango temporal que va desde hace ca. 5000 hasta I500 años AP y no habría coexistido con la práctica de inhumación en chenques (Zilio 2015). 
En cuanto a los concheros, constituyen el rasgo principal y dominante en Punta Medanosa, llegando incluso a determinar y condicionar la conservación de las dunas, ya que en algunos casos las densas lentes de valvas retrasan su deflación. De acuerdo con diversos estudios de distribución de concheros (por ej. Castro et al. 200I; Hammond et al. 2013; Hammond 2015; Zubimendi 2019a) y los resultados de la base de datos podemos postular que existen dos grandes concentraciones de este tipo de sitios: una ubicada entre la zona de Ensenada Ferrer y Roca del Cura; y la otra en el extremo noreste, conocido comúnmente por la gente de la zona como Punta Buque. Dispersiones menores -y según observaciones a priori, de menores densidades- existen entre estas dos últimas mencionadas y el extremo sudeste. Este tipo de sitios, que por sus características pueden ser interpretados como sitios residenciales donde se desarrollaron una variada gama de actividades (Hammond 2015; Zubimendi et al. 2005) y por las características estructurales de algunos de ellos, parecen reflejar momentos de ocupación relativamente prolongada, con una explotación intensiva de los recursos marinos como moluscos y pinnípedos. El rango cronológico de los sitios concheros abarca desde hace ca. 6300 a 360 años AP, lo que corresponde a gran parte del Holoceno medio y todo el tardío, incluyendo momentos postcontacto (Zubimendi 20rga), aunque conviene resaltar que no se han registrado en el campo ni en las colecciones privadas provenientes de Punta Medanosa restos materiales (como metales, gres, o vidrio; ver por ejemplo, Bianchi 2020) que pudieran reflejar relaciones de las poblaciones patagónicas con navegantes europeos o de momentos coloniales.

\subsection{CONTEXTOS HISTÓRICOS Y ALTERACIONES RECIENTES}

Es interesante el registro arqueológico histórico, compuesto exclusivamente por un pecio en el intermareal al norte de la isla Liebres -accesible en la máxima bajante- $y$ restos de naufragios que se hallan dispersos por buena parte del perímetro de dicha isla. Estos se hallan tanto en el intermareal, como la línea de playa, y algunos de ellos han sido transportados al interior de la isla, e incluso reciclados como parte de estructuras recientes (Zubimendi 2020). Otro pecio es observable en las bajantes excepcionales al sur de la isla, aunque el mismo no es accesible a pie. Los restos provendrían de estos naufragios, que según las escasas referencias que hemos conseguido, habrían encallado a fines del siglo XIX o comienzos del XX9 .

Mención aparte se merecen aquellos datos que reflejan alteraciones antrópicas recientes de distinto tipo (Figura 9), principalmente la construcción de nuevas estructuras de piedra, como pircados y fogones, en general cerca de caminos o huellas de vehículos. Punta Medanosa es una localidad arqueológica muy conocida en la costa norte de Santa Cruz para la práctica del coleccionismo, esto es, la

9. No sabemos a qué barcos pertenecen, pero uno de ellos podría tratarse del buque Comodoro Rivadavia de la Línea Nacional al Sud, que naufragó al sudoeste de isla Pingüino en el año 1901 (https://www.histarmar.com.ar/ Naufragios/Naufragios-SantaCruz/ComodoroRivadavia.htm, accedido 2 de marzo de 2021). 


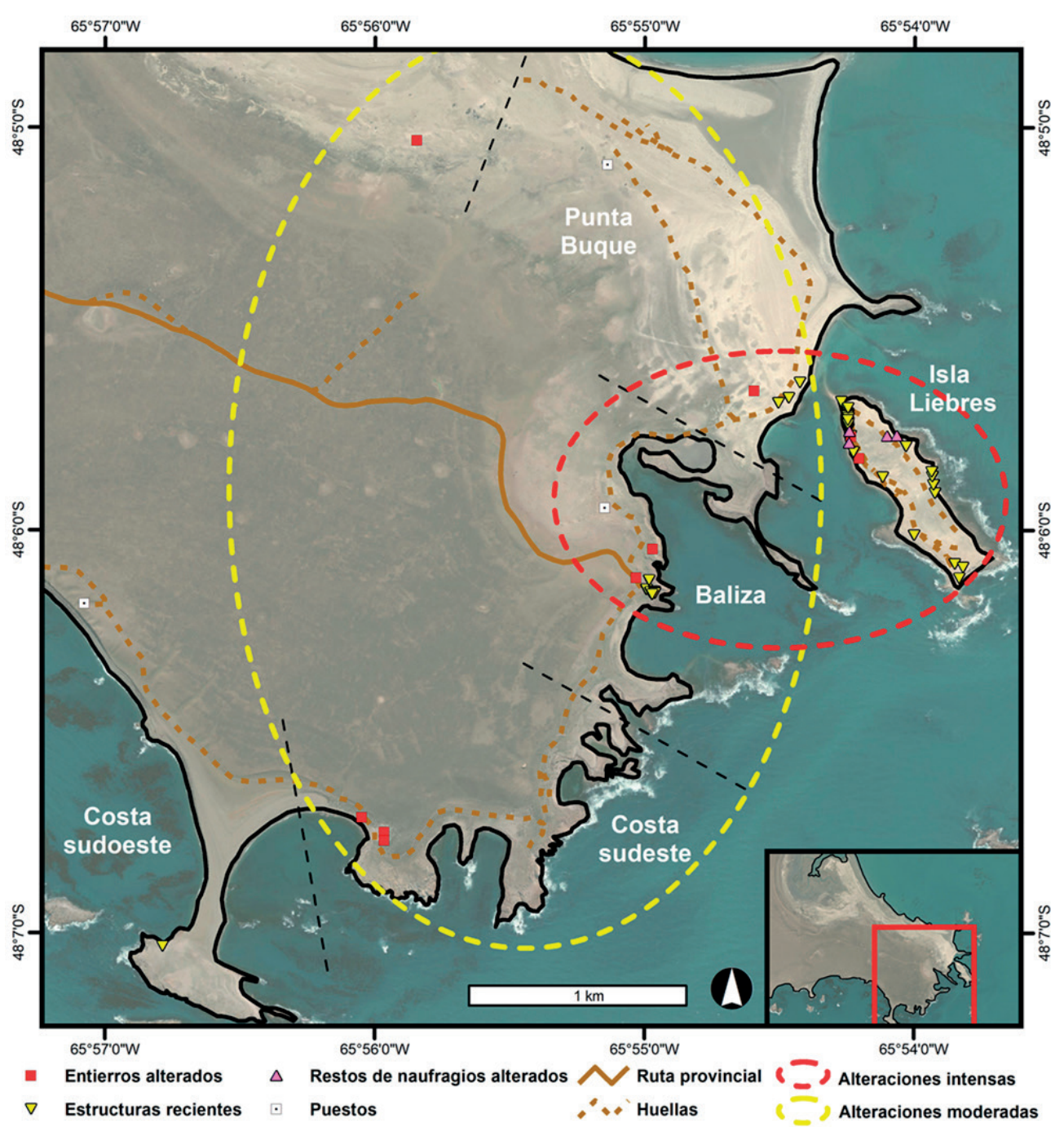

FIGURA 9. DETALLE DE LAS ALTERACIONES ANTRÓPICAS RECIENTES IDENTIFICADAS EN LOS SECTORES PUNTA BUQUE, ISLA LIEBRES, BALIZA Y COSTA SUDESTE. Figura elaboración propia

recolección de piezas arqueológicas por parte de particulares. Esta práctica es común al menos desde la década de I950, incluso se han publicado trabajos sobre piezas recolectadas por particulares (por ejemplo, Molina I976). Este tipo de alteraciones son muy difíciles, si no imposibles, de representar espacialmente. Sin embargo, en los últimos años y principalmente desde fines del año 20I2, se han registrado estructuras de piedra tipo pircados o fogones para hacer fuego y protegerse del viento. Estas se ubican en general cerca de la costa, y se concentran en los sectores Baliza e isla Liebres. En el primero, que es el sector más accesible al llegar a Punta Medanosa, ofrece los mejores lugares de reparo ante los fuertes vientos. En torno al afloramiento de más de $20 \mathrm{~m}$ de altura donde se halla La Baliza, y aprovechando las paredes rocosas, existen gran cantidad de estructuras de pircados y fogones. En sus cercanías hay basura de distinto tipo. En cuanto a isla Liebres, se accede con vehículos cuando baja la marea, y suele ser utilizada para realizar campings 
por parte de pescadores. Allí se han registrado una gran cantidad -creciente cada año- de estructuras de piedra recientes y basura. Estas estructuras construidas de forma asistemática y dispersas, en general, cerca de la costa, se ven acompañadas por gran cantidad de basura, así como en algunos casos la alteración de entierros (Zubimendi 2019b, 2020).

Otras estructuras que se han identificado en Punta Medanosa lo constituyen tres puestos de algueros ubicados en distintos sectores: Punta Buque, Baliza y Costa sudoeste. Se trata de construcciones precarias, construidas con materiales fácilmente desmontables (Ibarroule et al. 20II), por los dueños de la estancia durante la década de 1990 para el alojamiento de algueros durante las campañas de recolección de macroalgas de interés comercial (Piriz y Casas 1996). Esta actividad económica la desarrollaron a escala local durante dicha década y parte del 2000, aunque como emprendimiento económico no prosperó, por lo que fue abandonado. De acuerdo con las observaciones realizadas, el impacto en torno a los mismos es mínimo y localizado.

También se han registrado algunos casos de entierros alterados en distintas zonas de Punta Medanosa, no sólo en la costa. Algunos casos corresponden a restos humanos insertos en matriz de dunas, que probablemente al quedar expuestos por la erosión han sido removidos por visitantes ocasionales a la localidad. Entre estos se halla el sitio $\mathrm{PM}_{3}$ mencionado precedentemente, así como varios restos óseos encontrados insertos en una mata de calafate en el año 2000 , los cuales provendrían del entierro en médano El Amanecer i y 2 que se ubica a poca distancia (Zilio 2015, 20I6); y, por último, un fragmento proximal de peroné humano cerca de la baliza que, debido a su localización, interpretamos habría sido descontextualizado por algún turista (Zubimendi 2orgb). También se han registrado varios entierros de tipo chenque que han sido alterados, incluso para la construcción de pircados y fogones, principalmente en la isla Liebres (Zubimendi 20I9a), pero también en Punta Buque. Existe el antecedente del entierro en chenque alterado en el sector Baliza que Moreno sondeó en el año I99I (Moreno y Romagnoli I99I).

\subsection{HERRAMIENTA PARA LA GESTIÓN DEL PATRIMONIO}

En término generales, podemos zonificar las alteraciones antrópicas como intensas o potencialmente graves sobre el registro arqueológico en torno a la línea costera de la isla Liebres y la Baliza, así como también cerca de huellas de vehículos; y con un grado de riesgo moderado en los sectores Punta Buque y Costa sudeste (Figura 9). En la primera zona, los impactos antrópicos implican la construcción de nuevas estructuras, la contaminación mediante basura, así como en algunos casos la alteración de estructuras de entierro de tipo chenque. En la segunda, que se ubica en general a una mayor distancia de los caminos existentes y abarcando un espacio más amplio y de difícil delimitación, se han registrado principalmente restos humanos disturbados en médanos ${ }^{\text {10 }}$. Esta última zona es la que concentra la mayoría

10. En varias oportunidades se observaron gran cantidad de huellas de vehículos $4 \times 4$ por los médanos de estos 
de los sitios concheros, y donde se practica con mayor intensidad el coleccionismo de piezas arqueológica. En las restantes zonas no se han registrado estructuras recientes, sin embargo, no se puede descartar la práctica del coleccionismo, que constituye la alteración más común en Punta Medanosa, pero que no puede ser representada espacialmente, aunque se estima que la misma ha sido elevada al menos desde mediados del siglo XX (Zubimendi et al. 2015-2016).

Sin duda, la carta arqueológica generada constituye también una herramienta útil en la gestión del patrimonio arqueológico existente en Punta Medanosa. La diferenciación de los distintos tipos de sitios en determinadas zonas permite plantear la necesidad del cuidado y la protección de estos. Es especialmente importante la salvaguarda del registro mortuorio de esta localidad, la cual ha debido de ocupar un lugar de importancia para las poblaciones que habitaron esta parte del territorio por un amplio periodo de tiempo, que abarca el Holoceno medio y tardío (Zubimendi 20I9a). En este sentido, es interesante la coexistencia de diversas modalidades de entierro en la misma localidad y a lo largo del tiempo (Zilio 20I9), lo que constituye un ejemplo de gran interés a nivel regional ya que en un espacio relativamente acotado - unos pocos kilómetros cuadrados- presenta múltiples y variadas evidencias de las poblaciones humanas del pasado patagónico (Zubimendi 20I9a), una región que fue habitada por grupos cazadores recolectores de muy bajas densidades poblacionales $y$ alta movilidad (por ejemplo, Borrero 200I). Es por ello que esta carta podrá aportar a los diversos gestores responsables de la protección patrimonial, dado que el estado actual de desprotección en que se encuentra lo deja librado al abandono, destrucción y el coleccionismo; situación que queda especialmente patente en la isla Liebres (Zubimendi 2019b, 2020). El hecho de que recientemente parte de la localidad se encuentre dentro del Parque Interjurisdiccional Marino Isla Pingüino es auspicioso y permite augurarle un mejor futuro, no solo para detener las alteraciones que hemos registrado, sino incluso para iniciar políticas de gestión acordes a su importancia dentro de la arqueología patagónica. Esperamos que esta carta pueda ser un insumo para la gestión del patrimonio cultural arqueológico y modelo para otras partes del mismo parque.

\section{CONSIDERACIONES FINALES}

El ordenamiento y sistematización de la información espacial existente de Punta Medanosa constituye una línea de base de los trabajos realizados por el equipo de investigación Arqueología de la Costa Norte de Santa Cruz a lo largo de 30 años, en los cuales se integraron diferentes metodologías, objetivos y participaron distintas personas (sensu Mariano et al. 2014).

La base de datos creada con SIG permitirá realizar análisis estadísticos espaciales y modelados cuantitativos más profundos, ya que la cobertura de datos disponible

sectores, las cuales cruzaban por medio de los sitios, provocando la descontextualización de restos arqueológicos, y acelerando los procesos naturales de deflación. 
es suficientemente representativa del registro arqueológico de Punta Medanosa, al menos de las partes norte y este. Mientras que la costa sudoeste deberá ser parte de la agenda a futuro para poder relevar y obtener información espacial sobre el registro arqueológico que, potencialmente, podría existir en la misma. Dadas las características del registro arqueológico en esta localidad, como la alta densidad de distintos tipos de sitios y extensa secuencia de ocupación que abarca más de 6000 años, la información generada constituye un aporte valioso y de interés para definir futuras líneas de trabajo que permitirán conocer de mejor forma el paisaje de Punta Medanosa y por, sobre todo, los cambios que han ocurrido en la misma a lo largo del Holoceno.

Asimismo, este trabajo pretende ser también un punto de partida que permitirá una actualización constante en la base de datos (Magnin 20ı8; Panizza y Oliva 2018), así como avanzar en la estandarización y agilización de las consultas, la generación de cartografías temáticas y una mayor transferencia de información no solo para el desarrollo de investigaciones académicas o el intercambio con otros investigadores de la región, sino también para la gestión del patrimonio arqueológico y la definición de políticas de acción para su conservación (Lanzelotti et al. 2015; Martín Ruiz et al. I995-I996). En este sentido, constituye un aporte más a la búsqueda de recuperar y sistematizar información generada en el marco de proyectos de investigación

La Plata, II de agosto de 202I

\section{Agradecimientos}

Quisiera agradecer a quienes participaron en los trabajos de campo en Punta Medanosa y dejaron información que se ha podido volcar en este trabajo: Alicia Castro, Eduardo Moreno, Fernando Moreno, Victoria Díaz, Rocío Giménez, Laura Uehara, Fernando Pepe, María Andolfo, Karina Martinelli, Lucia Mazzitelli, Pablo Ambrústolo, Heidi Hammond, Laura Ciampagna, Leandro Zilio, Joaquín Rumbo y Carolina Contreras. También agradezco a Soledad Caracotche y Rocío Blanco por su aporte al estudio de las alteraciones recientes y el registro histórico de isla Liebres. Por otro lado, a lo largo de los años siempre hemos recibido el apoyo y ayuda de Sergio y Luli Vidal, dueños de El Amanecer, donde se halla Punta Medanosa. Los estudios se realizaron dentro del proyecto PICT 20I4-359I «Localidad arqueológica Punta Medanosa: cambios geomorfológicos y arqueológicos a lo largo del Holoceno medio y tardío». 


\section{REFERENCIAS CITADAS}

ApARICIO, F. I933-I935: «Viaje preliminar de exploración en el territorio de Santa Cruz». Publicaciones del Museo Antropológico y Etnográfico de la Facultad de Filosofía y Letras III: 7I-92.

AsSANDRI, S y GASTALDI, M. R. 20I8: «Cuarenta años de investigaciones: datos espaciales, arqueología y SIG en el Valle de Ambato (Provincia de Catamarca, Argentina)». Mundo de Antes I2(2): I3-4I.

Berón, M., Baffi, E., Molinari, R., Barrientos, G., Aranda, C. y Luna, L. 2000: «Estructuras funerarias de momentos tardíos en Pampa-Patagonia. El Chenque de Lihué Calel». En Desde el país de los Gigantes. Perspectivas arqueológicas en Patagonia, Tomo I. Universidad Nacional de la Patagonia Austral. Río Gallegos: I4I-I6o.

BiAnchi, P. E. 2020: «¿Y qué hacemos con las botellas? Raspadores de vidrio en inmediaciones de un puesto de estancia patagónico». La Zaranda de Ideas. Revista de Jóvenes Investigadores I8(I): 6-25.

Birabén, M. y Hylton Scott de Birabén, M. I. i936: «Viaje alrededor de Santa Cruz». Revista del Museo de La Plata, Sección Oficial, Nueva Serie: 93-I64.

Birabén, M. y Hylton Scott de Birabén, M. I. I939: «Observaciones sobre el pingüino Spbeniscus magellanicus (J. R. Forster)». Physis I6(48): 245-25I.

Borrero, L. A. 200i: El poblamiento de la Patagonia. Toldos, Milodones y Volcanes. Emecé. Buenos Aires.

CARRARA, I. S. I952: Lobos marinos, pingüinos y guaneras de las costas del litoral marítimo e islas adyacentes de la República Argentina. Informe técnico, Facultad de Ciencias Veterinarias, Universidad Nacional de La Plata. La Plata.

Castro, A. y Moreno, E. I988: Informe de los trabajos de campo en la costa norte de Santa Cruz. Manuscrito inédito.

Castro, A. y Moreno, E. 2000: «Noticia sobre enterratorios humanos en la costa Norte de Santa Cruz - Patagonia - Argentina». Anales del Instituto de la Patagonia, Serie Ciencias Humanas 28: 225-232.

Castro, A., Moreno, E., Andolfo, M. A. y Zubimendi, M. A. 20oi: «Distribución espacial de sitios en la localidad de Punta Medanosa». Relaciones de la Sociedad Argentina de Antropología XXVI: 303-322.

Castro, A., Moreno, E. y PeÑa, C. 2002: «Los enterratorios de la Localidad Punta Medanosa y Campo de Chenques: distribución y variabilidad». Trabajo presentado en Taller Internacional de Arqueología de Entierros Humanos en Fuego-Patagonia, Puerto Natales, Chile.

Castro, A. S., Moreno, E., Andolfo, M. A. Giménez, R., Peña, C., Mazzitelli, L., Zubimendi, M. A. y Ambrústolo, P. 2003: «Análisis distribucionales en la costa de Santa Cruz (Patagonia argentina): alcances y resultados». Anales del Instituto de la Patagonia, Serie Ciencias Humanas 31: 69-94.

Castro, A., Gómez Otero, J., Arrigoni, G. y Moreno, E. 2004: «Prospección macrorregional comparativa a las loberías de la costa atlántica continental de Patagonia: algunas claves sobre el uso del espacio y de otros recursos». En M. T. Civalero; P. M. Fernández; A. G. Guráieb (Eds.), Contra viento y marea. Arqueología de Patagonia. Instituto Nacional de Antropología y Pensamiento Latinoamericano. Buenos Aires: I97-215.

Castro, A., Zubimendi, M. A. y Ambrústolo, P. 20II: «Archaeological littoral sites on the northern coast of Santa Cruz: Valuable evidence of sea level changes on the continental 
Patagonian coasts (Argentina)». Quaternary International 245: III-I2I. https://doi. org/I0.IOI6/j.quaint.20I1.03.003

CiAmpagna, M. L. 2015: Estudio de la interacción entre grupos cazadores recolectores de Patagonia y las plantas silvestres: el caso de la costa norte de Santa Cruz durante el Holoceno medio y tardío. Tesis Doctoral, Facultad de Ciencias Naturales y Museo, Universidad Nacional de La Plata.

Ciampagna, M. L. 20I8: «Punzones de madera arqueológicos de Punta Medanosa (costa norte de Santa Cruz, Argentina)». Arqueología 24(I): 173-190. https://doi.org/I0.34096/ arqueologia.t24.nI.423I

Conolly, J. 2008: «Geographical Information Systems and Landscape Archaeology». En B. David; J. Thomas, Handbook of landscape archaeology. Left Coast Press. California: 583-595.

Constante, M. 200I: Geomorfología y geología de Ensenada Ferrer, Provincia de Santa Cruz. Tesis de Licenciatura. Departamento de Ciencias Geológicas, Universidad de Buenos Aires.

Cruz, I. 2004: «Tafonomía de huesos de aves en Punta Medanosa (Dpto. Puerto Deseado, Santa Cruz, Argentina)». En M. T. Civalero; P. M. Fernández; A. G. Guráieb (eds.), Contra viento y marea. Arqueología de Patagonia. Instituto Nacional de Antropología y Pensamiento Latinoamericano. Buenos Aires: 455-468.

CRUZ, I. 2007: «Avian taphonomy: observations at two Magellanic penguin (Spheniscus magellanicus) breeding colonies and their implications for the fossil record». Journal of Archaeological Science 34: I252-I261. https://doi.org/I0.1016/j.jas.2006.I0.016

De Feo, E., Moreno, E. y Magnin, L. 20I8: «Nota editorial al Dossier Herramientas analíticas para el estudio del paisaje en la Arqueología Argentina. Cruzando fronteras y tiempos». Arqueología 24(3): 7-I3. https://doi.org/I0.34096/arqueologia.t24.n3.54I6

Feruglio, E. I950: Descripción geológica de la Patagonia. Dirección General de Yacimientos Petrolíferos Fiscales. Buenos Aires.

Gallego Muñoz, P. A. 2013: Estrategias de integración del patrimonio cultural en el Ordenamiento del Territorio. Tesis de Magister, Universidad Nacional de Colombia.

García del Nodal, B., y García del NodAl, G. I62I: Relación diaria del reconocimiento del nuevo Estrecho de San Vicente y del de Magallanes. Fernando Correa Montenegro editor. Madrid.

GoÑI, R. A. y G. BARRIEntos. 200o: «Estudio de chenques en el lago Salitroso, Provincia de Santa Cruz». En Desde el país de los Gigantes. Perspectivas arqueológicas en Patagonia, Tomo I. Universidad Nacional de la Patagonia Austral. Río Gallegos: I6I-I75.

Graussac, P. I9I2: «Toponymie historique des cotes de la Patagonie». Anales de la Biblioteca VII: $287-425$.

HAMmOND, H. 2013: «Propuesta metodológica para el estudio de conjuntos malacológicos de sitios concheros: su aplicación en la costa norte de Santa Cruz (Patagonia Argentina)». La Zaranda de Ideas 9(2): 77-IO2.

Hammond, H. 2015: Sitios concheros en la costa norte de Santa Cruz: su estructura arqueológica y variabilidad espacial en cazadores recolectores patagónicos. Tesis Doctoral Facultad de Ciencias Naturales y Museo, Universidad Nacional de La Plata.

Hammond, H. 2019: «Distribuciones espaciales de concheros en la costa norte de Santa Cruz, Patagonia argentina: características del registro arqueológico y uso del espacio litoral por poblaciones cazadoras recolectoras». Comechingonia 22(2): 8I-III. https://doi. org/I0.37603/2250.7728.v22.n2.25588

Hammond, H. y Zubimendi, M. A. 20I3: «Estudio de la composición de sitios concheros en la Costa Norte de Santa Cruz (Patagonia Argentina)». En Zangrando, A. F.; Barberena, R.; Gil, A.; Neme, G.; Giardina, M.; Luna, L.; Otaola, C.; Paulides, S.; Salgán, L.; Tívoli, A. 
(eds.), Tendencias teórico metodológicas y casos de estudio en la Arqueología de la Patagonia. Museo de Historia Natural de San Rafael. Mendoza: 405-415.

Hammond, H., Zubimendi, M. A. y Zilio, L. 20I3: «Composición de concheros y uso del espacio: aproximaciones al paisaje arqueológico costero en Punta Medanosa». Anuario de Arqueología 5: 67-84.

Hammond, H., Zilio, L. y CaStro, A. 20i6: «Distribución, emplazamiento y procesos de formación del registro arqueológico en Punta Medanosa, costa norte de Santa Cruz». Intersecciones en Antropología 4: 6I-74.

Homar, A. y Guillermo, A. 2017: «Digitalización de cartografía arqueológica. El caso del curso medio del río Limay en el noroeste patagónico». Cuadernos del Instituto Nacional de Antropología y Pensamiento Latinoamericano - Series Especiales 4(3): I6-21.

ibarroule, A. M., Tagliorette, A., y Sampaoli, P. 20ir: Estancias del noreste de la provincia de Santa Cruz. Su historia y su Patrimonio en la primera mitad del siglo XX. Ediciones UNPAedita. Río Gallegos.

LANZELOTTI, S. 20I7: «Los Sistemas de Información Geográfica en la arqueología argentina». REDSociales. Revista del Departamento de Ciencias Sociales 4(5): I83-192.

LANZELOTTI, S. 2020: «Desde I870 hasta 2020: antecedentes y estado actual de la arqueología de la cuenca del río Luján a I50 años de sus inicios». Relaciones de la Sociedad Argentina de Antropología XLV(2): 355-376.

Lanzelotti, S. L., AcuÑa SuAReZ, G. E. y ArZani, H. 20I5: «El Ordenamiento territorial y la gestión del patrimonio cultural del partido de Mercedes, Buenos Aires, Argentina». REDSociales. Revista del Departamento de Ciencias Sociales 3(I): 200-213.

MAGNIN, L. 20I7: «SIG arqueológico del Parque Nacional Bosques Petrificados de Jaramillo (Provincia de Santa Cruz, Argentina)». Arqueología 23(I): 83-98. http://revistascientificas. filo.uba.ar/index.php/Arqueologia/article/view/3659

Mariano, C., Endere, M. L., Pedrotta, V. y Mariano, M. 2oi4: «Anatomía de un Sistema de Información Geográfica (SIG) para el patrimonio arqueológico del centro de la provincia de Buenos Aires». Comechingonia I8(2): 225-247. https://doi.org/I0.37603/2250.7728.vI8.n2.I8I62

Martín Ruiz, J. M., Sánchez Bandera, P. J. y Martín Ruiz, J. A. I995-I996: «La carta arqueológica como instrumento de investigación y gestión patrimonial. El caso del valle de Abdalajís, Málaga». Mainake XVII-XVIII: 243-260.

Medina, R., Aguirre, M., Codignotto, J., Richiano, S. y Mormeneo, L. 20I4: «Geoformas, malacofauna y evolución costera durante el Holoceno en Ensenada Ferrer (Santa Cruz, Patagonia, Argentina)». Revista de la Asociación Geológica Argentina 7I(I): 69-8I.

MeNGHin, O. F. A. y BóRmidA, M. s/f: Arqueología de la costa patagónica. Manuscrito inédito.

Molina, M. J. I967-I970: «Arpones monodentados de la Patagonia Meridional». Acta Præhistorica VIII/X: I73-I79.

Molina, M. J. 1976: Patagónica. Prehistoria, tradiciones y mitologías. Librería Ateneo Salesiano. Roma.

Moreno, E. I989-I99I: Informe preliminar de los trabajos de campo en la costa norte de Santa Cruz. Manuscrito inédito.

Moreno, E. 2009: Arqueología e etnohistoria de la Costa Patagónica Central en el Holoceno Tardío. Secretaría de Cultura del Chubut. Rawson.

Moreno, E. y Romagnino, A. I99i: Arqueología de la costa norte de Santa Cruz. Informe de trabajos de campo año I99I. Manuscrito inédito.

Panizza, M. C. y Oliva, C. 20I8: «Carta arqueológica de Puan (provincia de Buenos Aires): una herramienta para la investigación y la conservación del patrimonio». Revista de Antropología del Museo de Entre Ríos 4(2): 67-83. 
Panza, J., Márquez, M. y Godeas, M. I994: Descripción de la Hoja Geológica 4966-I y II, Bahía Laura, provincia de Santa Cruz. Dirección Nacional del Servicio Geológico. Buenos Aires.

Pastor, S., Murrieta Flores, M. y García SAnjuán, S. 20I3: «Los SIG en la arqueología de habla hispana. Temas, técnicas y perspectivas». Comechingonia I7(2): 9-29. https://doi. org/I0.37603/2250.7728.vI7.n2.I8I88

PAZ SoldÁN, M. I884: «Nomenclatura y ortografía geográfica en la República Argentina. Nomenclatura». La Nueva Revista de Buenos Aires XII: 43-55.

Piriz, M. L., y CASAS, G. I996: «Macroalgas de interés comercial en las costas del sur de Chubut y norte de Santa Cruz». Informes Técnicos del Plan de Manejo Integrado de la Zona Costera Patagónica 26.

Reyes, O. y Méndez, C. 20ıo: «Precisando la cronología para la inhumación tipo chenque, valle del río Cisne (Aisén, Chile), Patagonia Central». Magallania 38(2): I27-132.

Schiavini, A., Crespo, E. A. y Szapkievich, V. i999: «Estado de la población del lobo marino de un pelo (Otaria flavescens) en las provincias de Santa Cruz y Tierra del Fuego». Informes Técnicos del Plan de Manejo Integrado de la Zona Costera Patagónica, 40.

Schiavini, A., Yorio, P., Gandini, P, Raya Rey, A. y De Boerma, A. 2005: «Los pingüinos de las costas argentinas: estado poblacional y conservación». Hornero 2O(I): 5-23.

Vitores, M. 20I5: «Contribución a una carta arqueológica de la cuenca media del Limay». Arqueología 2I(2): 237-257. http://revistascientificas.filo.uba.ar/index.php/Arqueologia/article/ view $/ 3659$

ZANGRANDO, A. F. J. 20I8: «Shell middens and coastal archaeology in southern south America». En Smith, C. (ed.), Encyclopedia of Global Archaeology. Springer. Cham. https:// doi.org/Io.1007/978-3-319-5I726-I_3024-I

Zılıo, L. 20I3: «Chenques en Patagonia centro-meridional: análisis de los patrones de distribución espacio-temporales». Comechingonia I7(2): 237-254. https://doi. org/I0.37603/2250.7728.vI7.n2.18199

ZılıIO, L. 2015: Prácticas mortuorias en la costa norte de Santa Cruz: arqueología de sociedades cazadoras recolectoras en paisajes costeros de la Patagonia argentina. Tesis Doctoral, Facultad de Ciencias Naturales y Museo, Universidad Nacional de La Plata.

ZılıIO, L. 20I6: «Primer contexto mortuorio del Holoceno medio en la costa norte de Santa Cruz (Patagonia argentina)». Magallania 44(2): 219-224.

ZılıI, L. 20I7: «Primeras investigaciones sobre una estructura mortuoria singular de cazadores-recolectores en la Patagonia argentina: el entierro Shag». Arqueología Iberoamericana 33: 57-63. https://doi.org/I0.528I/zenodo.I3I9IO6

ZILIO, L. 20I9: «Coexistencia de tres modalidades de entierro humanas durante el Holoceno tardío en la costa norte de Santa Cruz: el caso de los entierros El Zanjón 3, Shag y El Amanecer». Comechingonia 22(2): 37-59. https://doi.org/IO.37603/2250.7728.v22.n2.25583

Zilio, L., Zubimendi, M. A. y Hammond, H. 20I3: «Chenques en un paisaje costero: análisis espacial de estructuras de entierro en Punta Medanosa». Anuario de Arqueología 5: 253-267.

Zilio, L., Hammond, H. y CAStro, A. 20I7: «Levantamiento planimétrico y análisis liquenométrico en el sitio Campo de Chenques, costa norte de Santa Cruz (Patagonia argentina)». Chungara 49(I): 65-80. http://dx.doi.org/I0.4067/S0717-73562017005000007

Zilio, L., BuUs, S. y Hammond, H. 20I8: «La colección arqueológica «Pedro Dade» del Museo de La Plata». Revista del Museo de La Plata 3(2):368-392. https://doi.org/10.24215/25456377eo65

Zubimendi, M. A. 20iga: «Cambios y continuidades a lo largo del Holoceno en el uso humano de la localidad arqueológica Punta Medanosa (Patagonia argentina)». Relaciones de la Sociedad Argentina de Antropología XLIV(I): I07-I29. 
Zubimendi, M. A. 20I9b: «Análisis de alteraciones antrópicas recientes y su impacto sobre el registro arqueológico en la isla Liebres (costa norte de Santa Cruz)». En J. Gómez Otero; A. Svoboda; A. Banegas (comps.), Arqueología de Patagonia: el pasado en las arenas. IDEAUS. Puerto Madryn: 455-467.

ZuBimend, M. A. 2020: «Estructuras de piedra e impacto antrópico reciente en isla Liebres (localidad arqueológica Punta Medanosa, costa norte de Santa Cruz)». Magallania 48(I): 65-9I.

Zubimendi, M. A., Castro, A. y Moreno, E. 2005: «Procesos de ocupación de la costa norte de Santa Cruz (Argentina): una síntesis». Relaciones de la Sociedad Argentina de Antropología XXX: 225-233.

Zubimendi, M. A. y BeretTA, M. 20I5: «Caracterización y análisis de las puntas de arpón de la Patagonia continental argentina». Relaciones de la Sociedad Argentina de Antropología $\mathrm{XL}(\mathrm{I}): 303-332$

Zubimendi, M. A., Ambrústolo, P., Zllıı, L. y CAStro, A. 20I5: «Continuity and discontinuity in the human use of the north coast of Santa Cruz (Patagonia Argentina) through its radiocarbon record». Quaternary International 356: I27-I46. https://doi.org/Io.IoI6/j. quaint.2014.09.035

Zubimendi, M. A., Hammond, H., Zilio, L., Ambrústolo, P. y Castro, A. 20I5-20I6: «Análisis de los agentes de alteración del registro arqueológico identificados en la costa norte de Santa Cruz (Patagonia argentina)». Anales de Arqueología y Etnología 70-71: 159-180. 